\title{
Instantons on conical half-flat 6-manifolds
}

\author{
Severin Bunk, ${ }^{a}$ Olaf Lechtenfeld, ${ }^{a, b}$ Alexander D. Popov ${ }^{a}$ and Marcus Sperling ${ }^{a}$ \\ ${ }^{a}$ Institut für Theoretische Physik, Leibniz Universität Hannover, \\ Appelstraße 2, 30167 Hannover, Germany \\ ${ }^{b}$ Riemann Center for Geometry and Physics, Leibniz Universität Hannover, \\ Appelstraße 2, 30167 Hannover, Germany \\ E-mail: Severin.Bunk@itp.uni-hannover.de, \\ Olaf .Lechtenfeld@itp.uni-hannover.de, \\ Alexander.Popov@itp.uni-hannover.de, \\ Marcus.Sperling@itp.uni-hannover.de
}

ABSTRACT: We present a general procedure to construct 6-dimensional manifolds with $\mathrm{SU}(3)$-structure from $\mathrm{SU}(2)$-structure 5-manifolds. We thereby obtain half-flat cylinders and sine-cones over 5-manifolds with Sasaki-Einstein SU(2)-structure. They are nearly Kähler in the special case of sine-cones over Sasaki-Einstein 5-manifolds. Both half-flat and nearly Kähler 6-manifolds are prominent in flux compactifications of string theory. Subsequently, we investigate instanton equations for connections on vector bundles over these half-flat manifolds. A suitable ansatz for gauge fields on these 6-manifolds reduces the instanton equation to a set of matrix equations. We finally present some of its solutions and discuss the instanton configurations obtained this way.

Keywords: Solitons Monopoles and Instantons, Flux compactifications, Differential and Algebraic Geometry

ArXiv EPRINT: 1409.0030 


\section{Contents}

1 Introduction 1

$2 \mathrm{SU}(2)$ - and SU(3)-structures in 5 and 6 dimensions 4

2.1 Sasakian structures 4

$2.2 \mathrm{SU}(2)$-structures in $d=5 \quad 4$

2.3 Example: the 5-sphere 6

$2.4 \mathrm{SU}(3)$-structures in $d=6 \quad 9$

3 Cylinders and sine-cones over 5-manifolds with SU(2)-structure 10

$\begin{array}{lll}3.1 & \text { Calabi-Yau metric cones } & 12\end{array}$

3.2 Kähler-torsion sine-cones 12

3.3 Nearly Kähler sine-cones 14

$\begin{array}{ll}3.4 \text { Half-flat cylinders } & 15\end{array}$

$\begin{array}{ll}3.5 & \text { Summary of cone constructions } \\ \end{array}$

4 Instantons on conical 6-manifolds $\quad \mathbf{1 6}$

4.1 Definition and reduction of instanton equations on conical 6-manifolds 16

$\begin{array}{lll}4.2 & \text { Remarks on the instanton equation } & 19\end{array}$

4.3 Instantons on nearly Kähler sine-cones 20

4.3.1 Matrix equations - Part I 20

4.3.2 Nearly Kähler canonical connection 23

4.3.3 Matrix equations - Part II 25

$\begin{array}{ll}\text { 4.3.4 Transfer of solutions } & 28\end{array}$

$\begin{array}{lll}4.4 & \text { Instantons on half-flat cylinders } & 29\end{array}$

$\begin{array}{lll}\text { 4.4.1 Matrix equations - Part I } & 29\end{array}$

4.4.2 Matrix equations - Part II 30

5 Conclusions $\quad 32$

\section{Introduction}

Solitons and instantons are important objects in modern field theory [1-3]. Solitons in supergravity theories are branes of various dimensions, which describe non-perturbative states of the underlying string theories or M-theory [4-6]. Branes in turn are sources of $p$-form flux fields. They can also wrap various supersymmetric cycles of special holonomy manifolds [6], and these cycles (which are calibrated submanifolds [7]) are defined, or calibrated, via the $p$-form fluxes. Thus, fluxes play an important role in the compactification of low-energy string theories and M-theory. 
String vacua with $p$-form fields along the extra dimensions ("flux compactifications") have been intensively studied in recent years (see [8,9] for a review and references). In particular, fluxes in heterotic string theories, which play a prominent role in string-theory model building due to the easy incorporation of the standard-model gauge group, have been considered e.g. in [10-23]. Heterotic flux compactifications have been known for quite some time, starting from refs. [24-27] in the mid-1980s. On Calabi-Yau manifolds the introduction of fluxes partially resolves the vacuum degeneracy problem by giving masses to problematic moduli, but they lead to non-integrable SU(3)-structures (i.e. with intrinsic torsion) on the internal compact 6-manifolds. Among these manifolds there are six-dimensional nearly Kähler and half-flat manifolds [10-14, 20-23].

Heterotic supergravity, as a low-energy effective field theory, preserves supersymmetry in 10 dimensions precisely if there exists at least one globally defined Majorana-Weyl spinor $\epsilon$ such that the supersymmetry variations of the fermionic fields (gravitino $\lambda$, dilatino $\phi$, and gaugino $\xi$ ) vanish, i.e. the so-called BPS equations

$$
\begin{aligned}
& \delta_{\lambda}=\nabla^{+} \epsilon=0, \\
& \delta_{\psi}=\gamma\left(\mathrm{d} \phi-\frac{1}{2} H\right) \epsilon=0, \\
& \delta_{\xi}=\gamma\left(\mathcal{F}_{\mathcal{A}}\right) \epsilon=0
\end{aligned}
$$

hold, wherein $\gamma(\omega)=\frac{1}{p !} \omega_{i_{1} \ldots i_{p}} \Gamma^{i_{1} \ldots i_{p}}$ is the Clifford map for a $p$-form $\omega$. The bosonic field content is given by the metric $g$, the dilaton $\phi$, the 3 -form $H$, and the gauge field $\mathcal{A}$. Further, $\nabla^{+}$is a metric compatible connection with torsion $H$.

The 10-dimensional space is assumed to be a product $\mathbb{M}^{p-1,1} \times M^{10-p}$, where $M^{10-p}$ is a $d=(10-p)$-dimensional internal manifold. Then (1.1a) translates into the existence of an covariantly constant spinor $\epsilon_{d}$ on $M^{d}$. Moreover, a globally defined non-vanishing spinor exists only on manifolds $M^{d}$ with reduced structure group (i.e. a $G$-structure), which in $d=6$ amounts to an $\mathrm{SU}(3)$-structure. Then a metric compatible connection, which leaves $\epsilon_{6}$ parallel and is also compatible with the $\mathrm{SU}(3)$-structure, always exists, but possibly has torsion. In other words, a connection with SU(3)-holonomy always exists on SU(3)manifolds. As a consequence, manifolds with special holonomy or $G$-structure are essential in string theory compactifications. Moreover, $G$-structures then allow for a $(d-4)$-form $\Psi$ on $M^{d}$, such that the natural choice for the 3 -form flux is $H=\star \mathrm{d} \Psi$.

In addition, the curvature $\mathcal{F}_{\mathcal{A}}$ of a connection $\mathcal{A}$ on a gauge bundle has to satisfy the generalized instanton equation (1.1c). In particular, the instanton equation can be introduced on any manifold with a $G$-structure. On manifolds $M^{d}$ with integrable $G$ structure, instantons have two crucial features. First, they solve the Yang-Mills equation (without torsion), and, second, the Levi-Civita connection on $T M^{d}$ already is an instanton.

The BPS equations (1.1) have to be supplemented by the $\alpha^{\prime}$-corrected Bianchi identity

$$
\mathrm{d} H=\frac{\alpha^{\prime}}{4}\left[\operatorname{tr}(R \wedge R)-\operatorname{tr}\left(\mathcal{F}_{\mathcal{A}} \wedge \mathcal{F}_{\mathcal{A}}\right)\right]
$$


due to the Green-Schwarz anomaly cancellation mechanism. Here $R$ is the curvature of a connection $\nabla$ on the tangent bundle. ${ }^{1}$ For compactifications with $\mathrm{d} H \neq 0$ one has the additional freedom to choose a gauge bundle compared to Calabi-Yau compactifications, wherein the vanishing of $\mathrm{d} H$ can be achieved by the "standard embedding" of the spin connection $\nabla^{+}$into the gauge connection $\mathcal{A}$, i.e. the gauge bundle is just $T M^{d}$. However, the choice of the gauge bundle for $\mathrm{d} H \neq 0$ is restricted by the Bianchi identity and the instanton equations (which on Kähler manifolds are just the Donaldson-Uhlenbeck-Yau equations [29-31] that correspond to a stability criterion on holomorphic bundles).

By a theorem of Ivanov [28], a solution to the BPS equations (1.1) and the Bianchi identity (1.2) satisfies the heterotic equations of motion if and only if the connection $\nabla$ is an $\mathrm{SU}(3)$-instanton in $d=6$. In other words, $R$ and $\mathcal{F}_{\mathcal{A}}$ are treated on the same footing in a pure supergravity view, i.e. $\gamma\left(\mathcal{F}_{\mathcal{A}}\right) \epsilon=\gamma(R) \epsilon=0$. Therefore, in the spirit of [22, 23, 32, 33], we study the instanton equation (1.1c) for non-integrable $\mathrm{SU}(3)$-structures in order to provide an important ingredient for full heterotic supergravity solutions. ${ }^{2}$

The construction of metric cones and sine-cones over manifolds $M^{d}$ with a $G$-structure provides a tool to generate and link different $G^{\prime}$-structures on $(d+1)$-dimensional manifolds. Most prominently, Sasaki-Einstein 5-manifolds generate a Calabi-Yau structure on their metric cone and a nearly Kähler structure on their sine-cone. A generalization is possible by means of the notion of hypo geometry, in particular to hypo, nearly hypo and double hypo $\mathrm{SU}(2)$-structures; see for instance [34]. Double hypo structures lift to nearly Kähler as well as to half-flat $\mathrm{SU}(3)$-structures on the sine-cone. The described "linking" phenomenon is well-known from the cases of cylinders, cones and sine-cones over nearly Kähler 6-manifolds, which lead to different $\mathrm{G}_{2}$-manifolds [35]. Here we use these techniques in order to construct 6-dimensional manifolds with special SU(3)-structures that may be valuable, for example, in flux compactifications of the heterotic string.

Supergravity in 10 dimensions allows for brane solutions which interpolate between an $\operatorname{Ad} S_{p+1} \times M^{9-p}$ near-horizon geometry and an asymptotic geometry $\mathbb{R}^{p-1,1} \times C\left(M^{9-p}\right)$, where $C\left(M^{9-p}\right)$ is a metric cone over $M^{9-p}$ (see e.g. [36, 37] and references therein). These brane solution in heterotic supergravity with Yang-Mills instantons on the metric cones $C\left(M^{9-p}\right)$ have been considered in $[22,23,38]$. Here, we take the first step to generalize them by considering sine-cones with nearly Kähler structures as well as cylinders with half-flat structures instead of metric cones with Kähler structures.

The generalization of Yang-Mills instantons to higher dimensions $(d>4)$ was first proposed in [39] and further studied in [29-31, 40-47] (see also references therein). Some solutions for $d>4$ have been found, namely $\operatorname{Spin}(7)$-instantons on $\mathbb{R}^{8}$ in $[48,49]$ and $\mathrm{G}_{2^{-}}$ instantons on $\mathbb{R}^{7}$ in [50-52]. For generic non-integrable $G$-structures, the instanton equation implies the Yang-Mills equation with torsion. However, as shown in [22], on manifolds with real Killing spinors the corresponding instantons solve the Yang-Mills equation without torsion even if the $G$-structure has non-vanishing intrinsic torsion. Recently, we constructed

\footnotetext{
${ }^{1}$ Different choices for $\nabla$, such as $\nabla^{+}$, are mentioned in [28].

${ }^{2}$ Choosing a different connection $\nabla$, for example $\nabla^{+}$, the BPS equations together with the Bianchi identity imply the heterotic equations of motion only up to higher $\alpha^{\prime}$-correction. This yields a perturbative solution in contrast to the exact solution advocated above.
} 
instantons on Kähler-torsion and hyper-Kähler-torsion sine-cones over Sasakian manifolds in [53]. In this paper we extend these studies to nearly Kähler sine-cones and half-flat cylinders over Sasaki-Einstein manifolds.

The outline of the paper is as follows: section 2 is devoted to a review of various $\mathrm{SU}(2)$-structures, focusing on hypo geometry and investigating the 5-sphere as an example. Section 3 then provides several cone constructions that link Sasaki-Einstein 5-manifolds to particular SU(3) 6-manifolds. In section 4 the instanton equations on these 6-dimensional $\mathrm{SU}(3)$-manifolds are derived, and utilizing a certain ansatz for the gauge connection these equations are reduced to matrix equations. We derive some particular solutions for these matrix equations by the choice of a suitable matrix ansatz and discuss their corresponding gauge field configurations.

\section{$2 \mathrm{SU}(2)$ - and SU(3)-structures in 5 and 6 dimensions}

\subsection{Sasakian structures}

We begin by introducing several geometric structures that will become important in the constructions of this paper. As in [54], an almost contact metric manifold is an odddimensional Riemannian manifold $\left(M^{2 m+1}, g\right)$ such that there exists a reduction of the structure group $\mathrm{SO}(2 m+1)$ of the bundle of orthonormal frames on $T M$ to $\mathrm{U}(m)$. For such manifolds there exist a 1-form $\eta$ and a 2-form $\omega$ such that $\eta \wedge(\omega)^{m} \neq 0$. Contact metric structures are characterized by $\mathrm{d} \eta=2 \omega$ in our sign convention.

An almost contact structure is characterized by the Nijenhuis torsion tensor [55] $\mathfrak{N}=$ $\left(\mathfrak{N}_{\mu \nu}^{\sigma}\right)$. A quasi-Sasakian structure is given by $\mathfrak{N}=0$ and $\mathrm{d} \omega=0$. In particular, if $\mathrm{d} \eta=\alpha \omega$ with $\alpha \in \mathbb{R}$, then the almost contact structure is called $\alpha$-Sasakian. If $\alpha=2$ the structure is called Sasakian.

Let us now specialize to the 5-dimensional case $M^{5}$, and let $e^{\mu}$ be an orthonormal coframe with $\mu=(a, 5)$ and $a=1, \ldots, 4$. Sasakian 5-manifolds are endowed with a 1-form $\eta$, 2-form $\omega, 3$-form $P$, and 4-form $Q$ satisfying the relations

$$
\begin{array}{rlrl}
\eta & =-e^{5}, & \omega & \left.\equiv \omega^{3}=\frac{1}{2} \eta_{a b}^{3} e^{a} \wedge e^{b}, \quad \eta\right\lrcorner \omega^{3}=0, \\
P & =\omega^{3} \wedge \eta, \quad Q=\frac{1}{2} \omega^{3} \wedge \omega^{3}, \\
\mathrm{~d} \eta & =2 \omega^{3}, \quad \mathrm{~d} P & =4 Q .
\end{array}
$$

Here $\eta_{a b}^{3}$ are the components of the self-dual 't Hooft tensors [1], and the contraction of two forms is defined as $\eta\lrcorner \omega:=*(\eta \wedge * \omega$ ) (see e.g. [56]).

\section{$2.2 \quad \mathrm{SU}(2)$-structures in $d=5$}

Let $M^{5}$ be 5-manifold with an $\mathrm{SU}(2)$-structure, i.e. the frame bundle of $M^{5}$ can be reduced to an $\mathrm{SU}(2)$ principal subbundle. It has been proven in [57] that an $\mathrm{SU}(2)$-structure is determined by a quadruplet $\left(\eta, \omega^{1}, \omega^{2}, \omega^{3}\right)$ of differential forms, wherein $\eta \in \Omega^{1}\left(M^{5}\right)$ and $\omega^{\alpha} \in \Omega^{2}\left(M^{5}\right)$ for $\alpha=1,2,3$. These forms satisfy

$$
\omega^{\alpha} \wedge \omega^{\beta}=2 \delta^{\alpha \beta} Q
$$

for the 4-form $Q=\frac{1}{2} \omega^{3} \wedge \omega^{3}$ with $\eta \wedge Q \neq 0$. 
Moreover, it has been shown in [57] that it is always possible to choose a local orthonormal basis $e^{1}, \ldots, e^{5}$ of forms on $M^{5}$ such that

$$
\eta=-e^{5}, \quad \omega^{1}=e^{23}+e^{14}, \quad \omega^{2}=e^{31}+e^{24}, \quad \omega^{3}=e^{12}+e^{34} .
$$

By means of the 't Hooft symbols $\eta_{a b}^{\alpha}$, one can express the 2-forms as

$$
\omega^{\alpha}=\frac{1}{2} \eta_{a b}^{\alpha} e^{a} \wedge e^{b}
$$

Here again $a, b=1,2,3,4$. Among the $\mathrm{SU}(2)$-structures in 5 dimensions there are several types having particularly interesting geometry. We will now recall their definitions following [34].

Sasaki-Einstein: a Sasaki-Einstein 5-manifold is a manifold carrying an SU(2)-structure defined by $\left(\eta, \omega^{1}, \omega^{2}, \omega^{3}\right)$, where these forms are subject to

$$
\mathrm{d} \eta=2 \omega^{3}, \quad \mathrm{~d} \omega^{1}=-3 \eta \wedge \omega^{2}, \quad \mathrm{~d} \omega^{2}=3 \eta \wedge \omega^{1} .
$$

Hypo: an SU(2)-structure on a 5-manifold is called hypo if

$$
\mathrm{d} \omega^{3}=0, \quad \mathrm{~d}\left(\omega^{1} \wedge \eta\right)=0, \quad \mathrm{~d}\left(\omega^{2} \wedge \eta\right)=0
$$

holds true. Hypo geometry, therefore, is a generalization of Sasaki-Einstein geometry.

Nearly hypo: an SU(2)-structure on a 5-manifold is called nearly hypo if it satisfies

$$
\mathrm{d} \omega^{1}=-3 \eta \wedge \omega^{2}, \quad \mathrm{~d}\left(\eta \wedge \omega^{3}\right)=2 \omega^{1} \wedge \omega^{1} .
$$

Note that any $\mathrm{SU}(2)$ structure which satisfies the first two identities of (2.5) is a nearly hypo structure.

Double hypo: an SU(2)-structure on a 5-manifold is called double hypo if it is hypo and nearly hypo simultaneously, i.e. if it satisfies (2.6) and (2.7). Thus, the Sasaki-Einstein 5 -manifolds are a subset of the double hypo manifolds.

As shown in [57], $\mathrm{SU}(2)$-structures in 5 dimensions always induce a nowhere-vanishing spinor on $M^{5}$. This will be generalized Killing if and only if the $\mathrm{SU}(2)$-structure is hypo, and Killing if and only if the SU(2)-structure is Sasaki-Einstein. In [22] it has been argued that in the latter case there exists a one-parameter family of metrics

$$
g_{M^{5}}=e^{2 h} \delta_{a b} e^{a} \otimes e^{b}+e^{5} \otimes e^{5}
$$

which is compatible with an $\mathfrak{s u}(2)$-valued connection on $M^{5}$ for which the Killing spinor is parallel. For the special value $\exp (2 h)=4 / 3$ the torsion of that connection is totally antisymmetric and parallel with respect to that connection, i.e. there exists a canonical $\mathfrak{s u}(2)$ connection. For all values of $h$ however, this connection is an $\mathfrak{s u}(2)$ instanton on $T M^{5}$ for the respective $\mathrm{SU}(2)$-structure. For $h=0, M^{5}$ is a Sasaki-Einstein manifold and the torsion components of the canonical connection read

$$
T^{a}=\frac{3}{4} P_{a \mu \nu} e^{\mu \nu} \quad \text { and } \quad T^{5}=P_{5 \mu \nu} e^{\mu \nu} .
$$




\subsection{Example: the 5-sphere}

We illustrate how different types of $\mathrm{SU}(2)$-structures are embedded into each other with the example of the 5 -sphere written as the homogeneous space $S^{5}=\mathrm{SU}(3) / \mathrm{SU}(2)$.

The SU(3)-structure constants can be chosen as

$$
\begin{aligned}
& f_{31}{ }^{6}=-f_{24}{ }^{6}={f_{23}}^{7}=-f_{14}{ }^{7}=f_{12}{ }^{8}=-f_{34}{ }^{8}=\frac{1}{2 \sqrt{3}}, \\
& f_{67}^{8}=f_{86}{ }^{7}=f_{78}{ }^{6}=\frac{1}{\sqrt{3}}, \\
& f_{12}{ }^{5}=f_{34}{ }^{5}=-\frac{1}{2},
\end{aligned}
$$

by using rescaled Gell-Mann matrices as a basis of $\mathfrak{s u}(3)$. The structure constants (2.10) are completely antisymmetric upon permutation of indices, and all other index combinations are zero. The Cartan-Killing form is then given by

$$
f_{A D}^{C} f_{C B}^{D}=\delta_{A B}, \quad A, B, C, D \in\{1,2, \ldots, 8\} .
$$

As local coframes $e^{\mu}=\left(e^{a}, e^{5}\right)$ on the coset space we use the images of the left-invariant 1 -forms on $\mathrm{SU}(3)$ under a pull-back along a section of the $\mathrm{SU}(2)$ principal bundle $\mathrm{SU}(3) \rightarrow$ $\mathrm{SU}(3) / \mathrm{SU}(2)$. The coset with the structure constants (2.10) is equipped with the CartanKilling metric, which can then be expressed as $(a, b, c, d=1, \ldots, 4$ and $i=6,7,8)$

$$
\begin{aligned}
& g_{a b}=f_{a d}{ }^{c} f_{c b}{ }^{d}+2 f_{a 5}{ }^{c} f_{c b}{ }^{5}+2 f_{a i}{ }^{c} f_{c b}{ }^{i}=\delta_{a b}, \\
& g_{55}=f_{5 d}{ }^{c} f_{c 5}{ }^{d}=\delta_{55} .
\end{aligned}
$$

The use of left-invariant objects on SU(3) enables us to explicitly compute connection components from the Maurer-Cartan equation. The connection 1-forms $\Gamma_{\nu}^{\mu}$ and the torsion 2 -forms $T^{\mu}$ are then given as

$$
\mathrm{d} e^{\mu}=-f_{i \nu}^{\mu} e^{i} \wedge e^{\nu}-\frac{1}{2} f_{\nu \sigma}^{\mu} e^{\nu} \wedge e^{\sigma}=-\Gamma_{\nu}^{\mu} \wedge e^{\nu}+T^{\mu},
$$

such that

$$
T^{\sigma}=\frac{1}{2} T_{\mu \nu}^{\sigma} e^{\mu} \wedge e^{\nu} \Rightarrow T_{\mu \nu}^{\sigma}=-f_{\mu \nu}^{\sigma} .
$$

With the Cartan-Killing metric (2.12) one obtains the totally antisymmetric components

$$
T_{a 5 b}=-f_{a 5 b}=f_{5 a b} .
$$

Note that

$$
\mathrm{d} e^{5}=-f_{a b}{ }^{5} e^{a} \wedge e^{b}=\frac{1}{2} \omega^{3},
$$

and that $\mathrm{SU}(3) / \mathrm{SU}(2)$ is endowed with an $\mathrm{SU}(2)$-structure given by $e^{5}$ and $\omega^{\alpha}$ as defined in (2.3). As a canonical connection on $\mathrm{SU}(3) / \mathrm{SU}(2)$ we have with the above choices

$$
{ }^{(c)} \Gamma_{b}^{a}=f_{i b}{ }^{a} e^{i}={ }^{(c)} \Gamma_{\mu b}^{a} e^{\mu} .
$$


Now, we introduce a two-parameter family of SU(2)-structures on $S^{5}$ by a rescaling of the $\mathfrak{s u}(3)$ generators. Consider

$$
I_{a} \rightarrow \tilde{I}_{a}=\frac{1}{\beta} I_{a}, \quad I_{5} \rightarrow \tilde{I}_{5}=\frac{1}{\gamma} I_{5}, \quad I_{i} \rightarrow \tilde{I}_{i}=I_{i}
$$

for $(\gamma, \beta) \in(\mathbb{R} \backslash\{0\}) \times \mathbb{R}^{+}$. (A change of sign for $\beta$ does not define a different $\mathrm{SU}(2)$ structure.) Consequently, the structure constants are changed as follows,

$$
\begin{array}{rlrl}
f_{5 b}{ }^{a} & \rightarrow \tilde{f}_{5 b}{ }^{a}=\frac{1}{\gamma} f_{5 b}{ }^{a}, & f_{a b}{ }^{5} \rightarrow \tilde{f}_{a b}{ }^{5}=\frac{\gamma}{\beta^{2}} f_{a b}{ }^{5}, \\
f_{a b}{ }^{i} & \rightarrow \tilde{f}_{a b}{ }^{i}=\frac{1}{\beta^{2}} f_{a b}{ }^{i}, & f_{i b}{ }^{a} \rightarrow \tilde{f}_{i b}{ }^{a}=f_{i b}{ }^{a}, \\
f_{j k}{ }^{i} \rightarrow \tilde{f}_{j k}{ }^{i} & =f_{j k}{ }^{i} .
\end{array}
$$

A rescaling of the generators of $\mathfrak{s u}(3)$ rescales the left-invariant vector fields and 1-forms accordingly, and this is propagated to the coset via the pullback as used before. In particular, the rescaled structure constants have to be used in the Maurer-Cartan equation in order to compute the differentials of the rescaled $\tilde{e}^{\mu}$. We can use (2.3) with respect to the new coframes $\tilde{e}^{\mu}$ to define a rescaled $\mathrm{SU}(2)$-structure on $S^{5}$. The differentials of the defining forms then read

$$
\begin{aligned}
\mathrm{d} \tilde{\eta} & =-\frac{\gamma}{2 \beta^{2}} \tilde{\omega}^{3}, \\
\mathrm{~d} \tilde{\omega}^{1} & =\frac{1}{\gamma} \tilde{\eta} \wedge \tilde{\omega}^{2}, \\
\mathrm{~d} \tilde{\omega}^{2} & =-\frac{1}{\gamma} \tilde{\eta} \wedge \tilde{\omega}^{1}, \\
\mathrm{~d} \tilde{\omega}^{3} & =0 .
\end{aligned}
$$

Thus, $\left(\tilde{\eta}, \tilde{\omega}^{1}, \tilde{\omega}^{2}, \tilde{\omega}^{3}\right)$ is a two-parameter family of hypo $\mathrm{SU}(2)$-structures on $S^{5}$, as the conditions (2.6) are satisfied for all values of $\beta$ and $\gamma$. For the value $(\gamma, \beta)=\left(-\frac{1}{3}, \frac{1}{2 \sqrt{3}}\right)$ this turns out to be nearly hypo additionally, and, as a consequence, at this value the $\mathrm{SU}(2)$ structure is double hypo. Furthermore, this particular SU(2)-structure is even SasakiEinstein, as we also show by a direct calculation of the Ricci tensor below. Therefore, the family of SU(2)-structures on $S^{5}$ does not discriminate between the double hypo and Sasaki-Einstein property. However, it shows how, by a simple rescaling of the generators of $\mathfrak{s u}(3)$, one can induce different $\mathrm{SU}(2)$-structure geometries on $S^{5}$.

Note that there are many possible choices of a Riemannian metric on the coset space. Among them are the Cartan-Killing metric and the round metric on $S^{5}$, which we consider in the following:

Cartan-Killing metric: from the definition (2.11) we obtain

$$
g_{\mathrm{CK}}=\delta_{a b} e^{a} \otimes e^{b}+e^{5} \otimes e^{5} .
$$


We express this with respect to local frames $\tilde{e}$ adapted to the Sasaki-Einstein SU(2)structure (i.e. for $\left.(\gamma, \beta)=\left(-\frac{1}{3}, \frac{1}{2 \sqrt{3}}\right)\right)$. Thus, we arrive at

$$
g_{\mathrm{CK}}=12 \delta_{a b} \tilde{e}^{a} \otimes \tilde{e}^{b}+9 \tilde{e}^{5} \otimes \tilde{e}^{5} .
$$

By means of the Maurer-Cartan equations

$$
\begin{aligned}
\mathrm{d} \tilde{e}^{\mu} & =-\frac{1}{2} \tilde{f}_{\nu \rho}{ }^{\mu} \tilde{e}^{\nu} \wedge \tilde{e}^{\rho}-\tilde{f}_{i \nu}{ }^{\mu} \tilde{e}^{i} \wedge \tilde{e}^{\nu} \\
\mathrm{d} \tilde{e}^{i} & =-\frac{1}{2} \tilde{f}_{j k}{ }^{i} \tilde{e}^{j} \wedge \tilde{e}^{k}-\frac{1}{2} \tilde{f}_{\mu \nu}{ }^{i} \tilde{e}^{\mu} \wedge \tilde{e}^{\nu}
\end{aligned}
$$

and demanding the torsion 2-form $T^{\mu}$ to vanish, one obtains

$$
{ }^{\mathrm{CK}} \Gamma_{b}^{a}=\tilde{f}_{i b}{ }^{a} \tilde{e}^{i}+\frac{1}{2} \tilde{f}_{c b}^{a} \tilde{e}^{c}
$$

for the connection 1-forms of the Levi-Civita connection induced by the Cartan-Killing metric on $S^{5}=\mathrm{SU}(3) / \mathrm{SU}(2)$. The curvature 2-form

$$
{ }^{\mathrm{CK}} R_{b}^{a}=\mathrm{d}^{\mathrm{CK}} \Gamma_{b}^{a}+{ }^{\mathrm{CK}} \Gamma_{c}^{a} \wedge{ }^{\mathrm{CK}} \Gamma_{b}^{c}
$$

can be computed, and all 2-form contributions proportional to $\tilde{e}^{j} \wedge \tilde{e}^{k}$ or $\tilde{e}^{j} \wedge \tilde{e}^{\mu}$ vanish due to the Jacobi identity [58]. Thus, the Ricci tensor reads

$$
\begin{aligned}
{ }^{\mathrm{CK}} \operatorname{Ric}_{a b} & =\tilde{f}_{a i}{ }^{c} \tilde{f}_{c b}{ }^{i}+\frac{1}{4}\left(\tilde{f}_{a c}{ }^{5} \tilde{f}_{5 b}{ }^{c}+\tilde{f}_{a 5}{ }^{c} \tilde{f}_{c b}{ }^{5}\right)=\frac{9}{2} \delta_{a b}, \\
{ }^{\mathrm{CK}} \mathrm{Ric}_{55} & =\frac{1}{4} \tilde{f}_{5 d}{ }^{c} \tilde{f}_{c 5}{ }^{d}=\frac{9}{4}, \quad{ }^{\mathrm{CK}} \operatorname{Ric}_{a 5}=0 .
\end{aligned}
$$

This shows that the choice of structure constants (2.10) yields an $\alpha$-Sasakian manifold with $\alpha=-\frac{1}{2}$ (cf. equation (2.20) for $\gamma=\beta=1$ ), but not an Einstein space.

Round metric: using again the local coframes $\tilde{e}$ adapted to the Sasaki-Einstein structure, the metric induced by stereographic projection from the ambient $\mathbb{R}^{6}$ reads

$$
g_{\mathrm{rnd}}=\delta_{a b} \tilde{e}^{a} \otimes \tilde{e}^{b}+\tilde{e}^{5} \otimes \tilde{e}^{5}=\delta_{\mu \nu} \tilde{e}^{\mu} \otimes \tilde{e}^{\nu} .
$$

Employing the Koszul formula for the round metric and the coframes $\tilde{e}^{\mu}$, one can calculate the Christoffel symbols of the Levi-Civita connection to be

$$
{ }^{\mathrm{rnd}} \Gamma_{\mu \nu}^{\rho}=\frac{1}{2} \tilde{f}_{\mu \nu}^{\rho}-2 \tilde{f}_{(\mu \nu)}^{\rho} .
$$

As before, the computation of the Ricci tensor is straightforward, and the result for this case is

$$
{ }^{\mathrm{rnd}} \operatorname{Ric}_{\mu \nu}=4\left(g_{\mathrm{rnd}}\right)_{\mu \nu}=4 \delta_{\mu \nu} .
$$

Hence, the 5-sphere endowed with the round metric is an Einstein space with Einstein constant 4 , just as expected. 


\section{$2.4 \mathrm{SU}(3)$-structures in $d=6$}

As pointed out in the introduction, one of our goals is the construction of $\mathrm{SU}(3)$-structures on 6-dimensional manifolds. Therefore, we introduce these structures and their characterization via intrinsic torsion classes. In a manner similar to subsection 2.2, an SU(3)-structure on a 6 -manifold $M^{6}$ is given by a reduction of the frame bundle to an $\mathrm{SU}(3)$ subbundle. An SU(3)-structure on a 6-dimensional manifold $M^{6}$ is characterized in terms of a triple $(J, \omega, \Omega)$, where $J$ is an almost complex structure, $\omega$ a $(1,1)$-form, and $\Omega$ a $(3,0)$-form with respect to $J$. These are subject to the algebraic relations

$$
\begin{aligned}
& \omega \wedge \Omega=0, \\
& \Omega \wedge \bar{\Omega}=-\frac{4 \mathrm{i}}{3} \omega \wedge \omega \wedge \omega .
\end{aligned}
$$

The compatible Riemannian metric is determined by $\omega(\cdot, \cdot)=g(J(\cdot), \cdot)$, and the $(3,0)$-form can be split into its real and imaginary part, i.e. $\Omega=\Omega^{+}+\mathrm{i} \Omega^{-}$. By an appropriate choice of a local frame, these forms can always be brought into the form

$$
\omega=e^{1} \wedge e^{2}+e^{3} \wedge e^{4}+e^{5} \wedge e^{6} \quad \text { and } \quad \Omega=\left(e^{1}+\mathrm{i} e^{2}\right) \wedge\left(e^{3}+\mathrm{i} e^{4}\right) \wedge\left(e^{5}+\mathrm{i} e^{6}\right) .
$$

For $\mathrm{SU}(3)$-structures in 6 dimensions, there exist several types of such structures with different geometric behavior, which is mostly governed by the differentials $\mathrm{d} \omega$ and $\mathrm{d} \Omega$. SU(3)-structures in 6 dimensions have been classified in terms of their five intrinsic torsion classes [59]. These are encoded in the differential of the defining forms in the following manner:

$$
\begin{aligned}
& \mathrm{d} \omega=\frac{3}{2} \operatorname{Im}\left(\left(W_{1}^{+}-\mathrm{i} W_{1}^{-}\right) \Omega\right)+W_{3}+W_{4} \wedge \omega, \\
& \mathrm{d} \Omega=\left(W_{1}^{+}+\mathrm{i} W_{1}^{-}\right) \omega \wedge \omega+\left(W_{2}^{+}+\mathrm{i} W_{2}^{-}\right) \wedge \omega+\Omega \wedge W_{5} .
\end{aligned}
$$

Here $W_{1}^{ \pm}$are real functions, $W_{4}$ and $W_{5}$ are real 1 -forms, $W_{2}^{ \pm}$are the real and imaginary part of a $(1,1)$-form, respectively, and $W_{3}$ is the real part of a $(2,1)$-form. Note that both $W_{2}$ and $W_{3}$ are primitive forms [8], i.e.

$$
\left.\omega\lrcorner W_{2}=0 \quad \text { and } \quad \omega\right\lrcorner W_{3}=0 .
$$

The Nijenhuis tensor gives rise to the components $W_{1}$ and $W_{2}$; thus, the almost complex structure $J$ of any $\mathrm{SU}(3)$-structure with non-vanishing $W_{1}$ or $W_{2}$ is non-integrable.

To finish this section, let us list the structures of particular relevance to us.

Kähler-torsion: on any almost Hermitian manifold $(M, g, J)$ there exists a unique connection preserving this structure and having totally antisymmetric torsion [60]. This connection is called the KT connection or Bismut connection [61]. Kähler-torsion (KT) 6manifolds are characterized by its torsion, which is given by

$$
T=J \mathrm{~d} \omega
$$


and which is the real part of a $(2,1)$-form. From [60] one can see that KT manifolds are complex manifolds, i.e. they enjoy

$$
W_{1}^{ \pm}=W_{2}^{ \pm}=0
$$

Note that in general their structure group is $\mathrm{U}(3)$ rather than $\mathrm{SU}(3)$, as they are a subclass of almost Hermitian structures. However, they may reduce to an $\mathrm{SU}(3)$-structure that is contained in the $\mathrm{U}(3)$-structure.

Calabi-Yau-torsion: if the KT connection is traceless, its holonomy is $\mathrm{SU}(3)$ instead of $\mathrm{U}(3)$ and, therefore, the structure group is reduced to $\mathrm{SU}(3)$. Conversely, if one is given an $\mathrm{SU}(3)$-structure $(g, \omega, \Omega)$ on $M^{6}$, this is always contained in the almost Hermitian structure defined by $(g, \omega)$. The KT connection of the latter then comprises an $\mathrm{SU}(3)$ connection for the $\mathrm{SU}(3)$-structure if and only if its $\mathrm{U}(1)$ part vanishes on the $\mathrm{SU}(3)$ subbundle. This can be written as a further condition on their torsion classes of the $\mathrm{SU}(3)$-structure under consideration (see, e.g. [62]), which reads

$$
2 W_{4}+W_{5}=0
$$

without restricting $W_{3}$. SU(3)-structures that are compatible with the KT connection of their almost Hermitian structure in this sense are called Calabi-Yau-torsion (CYT). Hence, CYT manifolds form a subset of KT manifolds, but with SU(3) structure group.

Nearly Kähler: an SU(3)-structure on a 6-manifold is nearly Kähler if

$$
W_{1}^{+}=W_{2}^{ \pm}=W_{3}=W_{4}=W_{5}=0 .
$$

Note that, in general, one does not need a vanishing $W_{1}^{+}$, but this can be achieved by suitable phase-transformation in $\Omega$.

Half-flat: an SU(3)-structure on a 6-manifold which satisfies

$$
W_{1}^{+}=W_{2}^{+}=W_{4}=W_{5}=0
$$

is called half-flat.

Note that generic nearly Kähler and half-flat 6-manifolds have a non-integrable almost complex structure $J$ and that nearly Kähler manifolds are a subclass of half-flat manifolds.

\section{Cylinders and sine-cones over 5-manifolds with SU(2)-structure}

Cylinders, metric cones, and sine-cones represent a tool for constructing $(n+1)$-dimensional $G^{\prime}$-structure manifolds starting from $n$-dimensional $G$-structure manifolds with $G \subset G^{\prime}$. At first, we review the well-known Calabi-Yau cone and the previously presented Kählertorsion sine-cone [53] for completeness. Next, we focus on the nearly Kähler sine-cone and the half-flat cylinder, which will provide the stage for the instanton equations considered in this paper. 
First, let us assume we are given a 5-dimensional manifold $M^{5}$ with an SU(2)-structure defined by $\left(\eta, \omega^{\alpha}\right)$ and a Riemannian metric $g_{5}$. These tensor fields induce global tensor fields on the Cartesian product $M \times I$, where $I$ is an interval. Due to the properties (2.3) of the $\mathrm{SU}(2)$-structure on $M^{5}$, around every point of $M \times I$, there is a local frame such that

$$
\eta=-e^{5}, \quad \omega^{\alpha}=\frac{1}{2} \eta_{a b}^{\alpha} e^{a} \wedge e^{b} \quad \text { and } \quad \mathrm{d} r=e^{6},
$$

if $r$ is the natural coordinate on the interval $I$. Next, we can apply transformations to these local frames; for example, perform a transformation like

$$
e^{\mu} \mapsto \phi(r) e^{\mu} \quad \text { and } \quad e^{6} \mapsto e^{6},
$$

changing the metric on $M^{5} \times I$ to the warped-product metric

$$
g=\mathrm{d} r^{2}+\phi(r)^{2} g_{5} \quad \text { on } M^{5} \times_{\phi} I
$$

Still, the forms $\left(\phi \eta, \phi^{2} \omega^{\alpha}, \mathrm{d} r\right)$ will have the same components as in (3.1) with respect to the altered frames.

Afterwards, one still has the freedom of further transformations. These need to map one $\mathrm{SU}(2)$-structure to another, which means that the defining forms need to have the standard components (2.3) with respect to the new frame. In addition, those transformations can be chosen to preserve the warped-product metric. In other words, these admissible transformations are given by maps from $M^{5} \times I$ to the normalizer subgroup of $\mathrm{SU}(2)$ in $\mathrm{GL}(6, \mathbb{R})$ (or $\mathrm{SO}(6)$ if one wants to preserve $g$ ), i.e.

$$
L: M^{5} \times I \rightarrow N_{\mathrm{GL}(6, \mathbb{R})}(\mathrm{SU}(2))
$$

The crucial statement is that if we are given a set of forms $\left(\eta, \omega^{\alpha}\right)$ on $M^{5} \times I$ such that around every point in $M^{5} \times I$ there is a local frame with respect to which (3.1) holds true, the forms defined by

$$
\begin{aligned}
\omega & =\omega^{3}-\eta \wedge \mathrm{d} r, \\
\Omega^{+} & =-\omega^{1} \wedge \mathrm{d} r+\omega^{2} \wedge \eta, \\
\Omega^{-} & =-\omega^{2} \wedge \mathrm{d} r-\omega^{1} \wedge \eta
\end{aligned}
$$

take the standard components (2.32) with respect to these local frames and, therefore, define an SU(3)-structure on $M^{5} \times I$. Note that $\omega$ and $\Omega$ are globally well-defined, simply because $\eta$ and the $\omega^{\alpha}$ are.

This provides us with a general way to construct $\mathrm{SU}(3)$-structure manifolds in 6 dimensions. Namely we push a given $\mathrm{SU}(2)$-structure on $M^{5}$ forward to $M^{5} \times I$ and apply transformations such that we still are given forms with components (3.1). Then we know that there exists an extension to an SU(3)-structure given by (3.4). In the following subsections we apply this procedure in several cases. 


\subsection{Calabi-Yau metric cones}

One result that makes Sasaki-Einstein manifolds interesting for string theorists as well as mathematicians is that their metric cones are Calabi-Yau. Here we demonstrate this explicitly for the 5-dimensional case. Consider a Sasaki-Einstein 5-manifold $M^{5}$ with local coframes $e^{\mu}$, where $\mu=(a, 5)$ and $a=1,2,3,4$. The metric on its metric cone reads

$$
g=r^{2}\left(\delta_{a b} e^{a} \otimes e^{b}+e^{5} \otimes e^{5}\right)+\mathrm{d} r \otimes \mathrm{d} r=r^{2}\left(\delta_{a b} e^{a} \otimes e^{b}+e^{5} \otimes e^{5}+e^{6} \otimes e^{6}\right)
$$

with

$$
e^{6}=\mathrm{d} \tau=\frac{\mathrm{d} r}{r} .
$$

The last equality in (3.5) displays the conformal equivalence to the cylinder over $M^{5}$ with the metric

$$
g_{\mathrm{cyl}}=\delta_{a b} e^{a} \otimes e^{b}+e^{5} \otimes e^{5}+e^{6} \otimes e^{6} .
$$

We can introduce an almost complex structure $J$ on the metric cone via

$$
J \hat{\Theta}^{\alpha}=\mathrm{i} \hat{\Theta}^{\alpha} \quad \text { for } \quad \alpha=1,2,3 \text { with } \hat{\Theta}^{\alpha}=\hat{e}^{2 \alpha-1}+\mathrm{i} \hat{e}^{2 \alpha},
$$

and we set $\hat{e}^{\hat{\mu}}=r e^{\hat{\mu}}$ for $\hat{\mu}=1, \ldots, 6$. The $\mathrm{SU}(3)$-structure forms $(\hat{\omega}, \hat{\Omega})$ have the local expressions

$$
\begin{aligned}
& \hat{\omega}=\hat{e}^{1} \wedge \hat{e}^{2}+\hat{e}^{3} \wedge \hat{e}^{4}+\hat{e}^{5} \wedge \hat{e}^{6}=r^{2}\left(\omega^{3}+e^{5} \wedge e^{6}\right), \\
& \hat{\Omega}=\hat{\Theta}^{1} \wedge \hat{\Theta}^{2} \wedge \hat{\Theta}^{3}
\end{aligned}
$$

for which a direct computation yields

$$
\mathrm{d} \hat{\omega}=0 \quad \text { and } \quad \mathrm{d} \hat{\Omega}=0 .
$$

Therefore, the metric cone introduced in (3.5) is indeed Calabi-Yau as all SU(3)-torsion classes vanish.

\subsection{Kähler-torsion sine-cones}

Consider a Sasaki-Einstein 5-manifold $M^{5}$ and the product manifold $M^{6}=M^{5} \times(0, \Lambda \pi)$ with the metric

$$
\begin{aligned}
g & =\Lambda^{2} \sin ^{2} \varphi\left(\delta_{a b} e^{a} \otimes e^{b}+e^{5} \otimes e^{5}\right)+\mathrm{d} r \otimes \mathrm{d} r \\
& =\Lambda^{2} \sin ^{2} \varphi\left(\delta_{a b} e^{a} \otimes e^{b}+e^{5} \otimes e^{5}+e^{6} \otimes e^{6}\right),
\end{aligned}
$$

where

$$
\varphi=\frac{r}{\Lambda} \quad \text { and } \quad e^{6}=\mathrm{d} \tau=\frac{\mathrm{d} \varphi}{\sin \varphi}
$$


and $\Lambda \in \mathbb{R}^{+}$is a scaling parameter. Equation (3.11b) shows that the metric on the sine-cone is conformally equivalent to the metric (3.7) on the cylinder over $M^{5}$.

The explicit solution of $\tau=\tau(\varphi)$ is computed to

$$
\tau=\ln \left|\tan \frac{\varphi}{2}\right|+\text { constant }
$$

and the integration constant can be chosen such that the sine-cone becomes the metric cone in the limit $\Lambda \rightarrow \infty$. Hence, the computation yields

$$
\tau(\varphi)=\ln \left(2 \Lambda \tan \frac{\varphi}{2}\right)=\ln \left(2 \Lambda \sqrt{\frac{1-\cos \varphi}{1+\cos \varphi}}\right) .
$$

Next, we introduce an almost complex structure $J$ and the associated fundamental $(1,1)$-form $\tilde{\omega}$ on the sine-cone as follows $(\alpha=1,2,3)$ :

$$
\begin{aligned}
& J \tilde{\Theta}^{\alpha}=\mathrm{i} \tilde{\Theta}^{\alpha} \quad \text { with } \tilde{\Theta}^{\alpha}=\Lambda \sin \varphi\left(e^{2 \alpha-1}+\mathrm{i} e^{2 \alpha}\right), \\
& J \tilde{\Theta}^{\bar{\alpha}}=-\mathrm{i} \tilde{\Theta}^{\bar{\alpha}} \quad \text { with } \quad \tilde{\Theta}^{\bar{\alpha}}=\overline{\tilde{\Theta}^{\alpha}}, \\
& \tilde{\omega}=\Lambda^{2} \sin ^{2} \varphi\left(\omega^{3}+e^{5} \wedge e^{6}\right),
\end{aligned}
$$

where $\omega^{3}$ is defined in (2.3). As shown in [53], the above structure comprises a Kählertorsion structure on the sine-cone. That is, there exists the uniquely defined Bismut $\nabla^{B}$ connection, which preserves $g$ and $J$, and has torsion given by

$$
T^{B}=J \mathrm{~d} \tilde{\omega} .
$$

Remarks: one can also introduce a globally well-defined complex (3,0)-form $\tilde{\Omega}$ defined as

$$
\tilde{\Omega}=\tilde{\Theta}^{1} \wedge \tilde{\Theta}^{2} \wedge \tilde{\Theta}^{3}=\Lambda^{3} \sin (\varphi)^{3}\left(\omega^{2}-\mathrm{i} \omega^{1}\right) \wedge \eta-\Lambda^{2} \sin (\varphi)^{2}\left(\omega^{1}+\mathrm{i} \omega^{2}\right) \wedge \mathrm{d} r .
$$

Applying the exterior differential yields

$$
\begin{aligned}
& \mathrm{d} \tilde{\omega}=2 \frac{\cos \varphi-1}{\Lambda \sin \varphi} \tilde{\omega} \wedge \tilde{e}^{6}=-\frac{2}{\Lambda} \tan \frac{\varphi}{2} \tilde{\omega} \wedge \tilde{e}^{6}, \\
& \mathrm{~d} \tilde{\Omega}=3 \frac{1-\cos \varphi}{\Lambda \sin \varphi} \tilde{\Omega} \wedge \tilde{e}^{6}=\frac{3}{\Lambda} \tan \frac{\varphi}{2} \tilde{\Omega} \wedge \tilde{e}^{6},
\end{aligned}
$$

thus rendering the sine-cone over $M^{5}$ an $\mathrm{SU}(3)$-structure manifold as defined in section 2.4. From (3.18) we immediately see that $J$ is integrable and

$$
2 W_{4}+W_{5}=-\frac{1}{\Lambda} \tan \frac{\varphi}{2} \tilde{e}^{6} \neq 0 \text { for } \Lambda<\infty,
$$

whence the Bismut connection does not preserve the $\mathrm{SU}(3)$-structure unless $\Lambda=\infty$. Nevertheless, the condition $3 W_{4}+2 W_{5}=0$ is satisfied, which is in agreement with the conformal equivalence between the sine-cone over a Sasaki-Eintein 5-manifold and the Calabi-Yau metric cone over $M^{5}[59,63]$. That is, the conformal equivalence of the Calabi-Yau cone and the Kähler torsion sine-cone also maps their two SU(3)-structures onto one another. We also note that $2 W_{4}+W_{5} \rightarrow 0$ as $\Lambda \rightarrow \infty$, and the KT sine-cone becomes the Calabi-Yau metric cone. Recall from section 2.4 that Kähler-torsion structures are U(3)-structures, whence one has to distinguish between this and the additional SU(3)-structure. 


\subsection{Nearly Kähler sine-cones}

In [34] a nearly Kähler structure on the sine-cone over a Sasaki-Einstein 5-manifold has been obtained by means of flow equations. Here, in contrast, we show that this structure can be constructed by means of a combined rotation and rescaling of the coframes of the cylinder over the Sasaki-Einstein 5-manifold. We will carry this construction out in the following three steps:

1. An SU(3)-structure on the cylinder over a Sasaki-Einstein 5-manifold $M^{5}$ can be introduced via a metric (3.7), an almost complex structure $J$ or the equivalent $(1,1)$ form $\omega$, and a $(3,0)$-form $\Omega$. These objects are

$$
\begin{aligned}
\omega & =\omega^{3}+e^{5} \wedge e^{6}=e^{1} \wedge e^{2}+e^{3} \wedge e^{4}+e^{5} \wedge e^{6}, \\
J \Theta^{\alpha} & =\mathrm{i} \Theta^{\alpha} \quad \text { for } \quad \Theta^{\alpha}=e^{2 \alpha-1}+\mathrm{i} e^{2 \alpha} \quad \text { with } \quad \alpha=1,2,3, \\
\Omega & =\Theta^{1} \wedge \Theta^{2} \wedge \Theta^{3}=-\omega^{2} \wedge e^{5}-\omega^{1} \wedge e^{6}+\mathrm{i}\left(\omega^{1} \wedge e^{5}-\omega^{2} \wedge e^{6}\right) .
\end{aligned}
$$

2. Next, we consider an $\mathrm{SO}(5)$-rotation of the $\mathrm{SU}(2)$-structure $\left(\eta, \omega^{\alpha}\right)$ on $M^{5}$. Let $\eta^{2}$ be the matrix of the 't Hooft symbols $\eta_{a b}^{2}$ and perform a rotation of the basis 1-forms $e^{1}, \ldots, e^{4}$,

$$
E=\left(\begin{array}{l}
e^{1} \\
e^{2} \\
e^{3} \\
e^{4}
\end{array}\right) \mapsto E_{\varphi}=\exp \left(\frac{\varphi}{2} \eta^{2}\right) E=\left(\begin{array}{cccc}
\cos \frac{\varphi}{2} & 0 & -\sin \frac{\varphi}{2} & 0 \\
0 & \cos \frac{\varphi}{2} & 0 & \sin \frac{\varphi}{2} \\
\sin \frac{\varphi}{2} & 0 & \cos \frac{\varphi}{2} & 0 \\
0 & -\sin \frac{\varphi}{2} & 0 & \cos \frac{\varphi}{2}
\end{array}\right)\left(\begin{array}{l}
e^{1} \\
e^{2} \\
e^{3} \\
e^{4}
\end{array}\right) .
$$

In the rotated frame $\left(e_{\varphi}^{a}, e^{5}\right)$ we define the $\mathrm{SU}(3)$-structure forms to have the same components as in the unrotated frame (3.20), i.e.

$$
\begin{aligned}
& \omega_{\varphi}=\omega_{\varphi}^{3}+e^{5} \wedge e^{6}, \\
& \Omega_{\varphi}=-\omega_{\varphi}^{2} \wedge e^{5}-\omega_{\varphi}^{1} \wedge e^{6}+\mathrm{i}\left(\omega_{\varphi}^{1} \wedge e^{5}-\omega_{\varphi}^{2} \wedge e^{6}\right),
\end{aligned}
$$

where $\omega_{\varphi}^{\alpha}=\frac{1}{2} \eta_{\mu \nu}^{\alpha} e_{\varphi}^{\mu \nu}$. Note that this is still an $\mathrm{SU}(3)$-structure on the cylinder, because the defining forms still have the standard components (3.20) with respect to the coframes $e_{\varphi}^{\mu}$.

3. Last, the pullback to the sine-cone $C_{s}\left(M^{5}\right)$ along the map establishing the conformal equivalence to the cylinder yields

$$
\begin{aligned}
e_{s}^{a} & =\Lambda e_{\varphi}^{a} \sin \varphi, \quad e_{s}^{5}=\Lambda e^{5} \sin \varphi, \quad e_{s}^{6}=\Lambda e^{6} \sin \varphi=\Lambda \mathrm{d} \varphi=\mathrm{d} r \\
\omega_{s}^{\alpha} & =\Lambda^{2} \omega_{\varphi}^{\alpha} \sin ^{2} \varphi, \quad \omega_{s}=\omega_{s}^{3}+\Lambda^{2} e^{5} \wedge e^{6} \sin ^{2} \varphi \\
\Omega_{s} & =\Lambda^{3} \Omega_{\varphi} \sin ^{3} \varphi
\end{aligned}
$$

as an $\mathrm{SU}(3)$-structure on the sine-cone. By a direct calculation we obtain

$$
\begin{aligned}
\mathrm{d} \omega_{s} & =-\frac{3}{\Lambda} \Omega_{s}^{+}, \\
\mathrm{d} \Omega_{s}^{+} & =0, \quad \mathrm{~d} \Omega_{s}^{-}=\frac{2}{\Lambda} \omega_{s} \wedge \omega_{s},
\end{aligned}
$$

which confirms that (3.23) induces a nearly Kähler structure on the sine-cone. 
Remarks: in the limit $\Lambda \rightarrow \infty$, in which the sine-cone becomes the metric cone, this nearly Kähler structure on the sine-cone is smoothly deformed to the Calabi-Yau structure on the metric cone since

$$
\lim _{\Lambda \rightarrow \infty} \mathrm{d} \omega_{s}=0 \quad \text { and } \quad \lim _{\Lambda \rightarrow \infty} \mathrm{d} \Omega_{s}=0 .
$$

Generically, the sine-cone, as a conifold, has two singularities at $\varphi=0$ and $\varphi=\pi$. As we see from (3.23), the SU(3)-structure cannot be extended to the tips, because all defining forms vanish at these points. Hence, the sine-cone is a nearly Kähler manifold only for $\varphi \in(0, \pi)$, and one cannot add the singular points.

\subsection{Half-flat cylinders}

Consider a 5-dimensional manifold $M^{5}$ endowed with a Sasaki-Einstein SU(2)-structure defined by $\left(\eta, \omega^{1}, \omega^{2}, \omega^{3}\right)$ as in section 2 . For an arbitrary coframe $e^{\mu}$ belonging to the $\mathrm{SU}(2)$-structure, consider the transformation

$$
\begin{array}{lll}
e_{z}^{1}=e^{4} \cos \zeta+e^{3} \sin \zeta, & e_{z}^{2}=-e^{1}, \\
e_{z}^{3}=e^{2}, & e_{z}^{4}=e^{3} \cos \zeta-e^{4} \sin \zeta \\
e_{z}^{5}=\varrho e^{5} & &
\end{array}
$$

Here $\zeta \in[0,2 \pi]$ and $\rho \in \mathbb{R}^{+}$are two constant parameters. For $\varrho=1$ this can be seen to be an $\mathrm{SO}(5)$-transformation of the coframe, such that the metric on $M^{5}$ is unchanged. Nevertheless, we obtain a two-parameter family of $\mathrm{SU}(2)$-structures on $M^{5}$ by defining

$$
\eta_{z}=\varrho \eta, \quad \omega_{z}^{\alpha}=\frac{1}{2} \eta_{\mu \nu}^{\alpha} e_{z}^{\mu} \wedge e_{z}^{\nu}, \quad g_{z}=\delta_{\mu \nu} e_{z}^{\mu} \otimes e_{z}^{\nu}
$$

These are globally well-defined as can be seen from

$$
\begin{aligned}
& \omega_{z}^{1}=-\omega^{3}, \\
& \omega_{z}^{2}=\omega^{1} \sin \zeta+\omega^{2} \cos \zeta, \\
& \omega_{z}^{3}=\omega^{1} \cos \zeta-\omega^{2} \sin \zeta,
\end{aligned}
$$

and, thus, yield a two-parameter family of $\mathrm{SU}(2)$-structures on $M^{5}$. Note that these structures are neither hypo nor nearly hypo any more.

With these $\mathrm{SU}(2)$-structures on $M^{5}$ at hand we define a two-parameter family of $\mathrm{SU}(3)$-structures on the metric cylinder $\left(M^{5} \times \mathbb{R}, \bar{g}_{z}=g_{z}+\mathrm{d} r \otimes \mathrm{d} r\right)$ by

$$
\begin{aligned}
\omega_{z} & =\omega_{z}^{3}-\eta_{z} \wedge \mathrm{d} r=\omega^{1} \cos \zeta-\omega^{2} \sin \zeta-\varrho \eta \wedge \mathrm{d} r \\
\Omega_{z}^{+} & =-\omega_{z}^{1} \wedge \mathrm{d} r+\omega_{z}^{2} \wedge \eta_{z}=\varrho\left(\omega^{1} \sin \zeta+\omega^{2} \cos \zeta\right) \wedge \eta+\omega^{3} \wedge \mathrm{d} r, \\
\Omega_{z}^{-} & =-\omega_{z}^{2} \wedge \mathrm{d} r-\omega_{z}^{1} \wedge \eta_{z}=-\left(\omega^{1} \sin \zeta+\omega^{2} \cos \zeta\right) \wedge \mathrm{d} r+\varrho \omega^{3} \wedge \eta
\end{aligned}
$$

which yields a two-parameter family of half-flat SU(3)-structures. The non-vanishing torsion classes can be computed to read

$$
\begin{aligned}
W_{1}^{-} & =\frac{3+2 \varrho^{2}}{3 \varrho}, \quad W_{2}^{-}=\frac{4 \varrho^{2}-3}{3 \varrho}\left(\omega_{z}^{3}+2 \eta_{z} \wedge \mathrm{d} r\right) \quad \text { and } \\
W_{3} & =\frac{2 \varrho^{2}-3}{2 \varrho}\left(\omega_{z}^{1} \wedge \mathrm{d} r+\omega_{z}^{2} \wedge \eta_{z}\right) .
\end{aligned}
$$




\begin{tabular}{|l|l|l|l|}
\hline Structure on $\boldsymbol{M}^{\mathbf{5}}$ & Cone construction & Structure on $\boldsymbol{M}^{\mathbf{6}}$ & Non-zero torsion classes \\
\hline \multirow{3}{*}{ Sasaki-Einstein } & cone & Calabi-Yau & -- \\
\cline { 2 - 4 } & sine-cone & Kähler-torsion & \\
\cline { 2 - 4 } & sine-cone with rotation & nearly Kähler & $W_{1}^{-}$ \\
\cline { 2 - 4 } & cylinder with rotation & half-flat & $W_{1}^{-}, W_{2}^{-}, W_{3}$ \\
\hline
\end{tabular}

Table 1. Summary of cone constructions linking Sasaki-Einstein to U(3) or SU(3)-structures in $d=6$ and the non-zero torsion classes for the respective $\mathrm{SU}(3)$-structures.

Furthermore, the conditions $\left.\omega_{z}\right\lrcorner W_{2}^{-}=0$ and $\left.\omega_{z}\right\lrcorner W_{3}=0$ are satisfied for any values of the parameters $\zeta$ and $\varrho$.

\subsection{Summary of cone constructions}

The different cone constructions linking Sasaki-Einstein to U(3) or SU(3) 6-manifolds, which have been presented in [53] and this paper, are summarized in table 1.

\section{Instantons on conical 6-manifolds}

\subsection{Definition and reduction of instanton equations on conical 6-manifolds}

Having constructed several 6-dimensional SU(3) manifolds in the last section, we now turn our attention to instanton equations on such spaces. Thus, let $M^{6}$ be a 6 -manifold with a connection $\mathcal{A}$ on the tangent bundle. The curvature 2 -form $\mathcal{F}$ associated to $\mathcal{A}$ is given by

$$
\mathcal{F}=\mathrm{d} \mathcal{A}+\mathcal{A} \wedge \mathcal{A}=: D_{\mathcal{A}} \mathcal{A}
$$

where $D_{\mathcal{A}}$ is the covariant differential associated to $\mathcal{A}$, and the Bianci identity $D_{\mathcal{A}} \mathcal{F}=0$ holds true. As before, we can perform the type-decomposition of a form with respect to any almost complex structure $J$, yielding

$$
\mathcal{F}=\mathcal{F}^{2,0}+\mathcal{F}^{1,1}+\mathcal{F}^{0,2} .
$$

For a given $\mathrm{SU}(3)$-structure $(\omega, \Omega)$ on a 6 -manifold and a curvature 2 -form $\mathcal{F}$, the instanton equation can be defined in two steps: first, the pseudo-holomorphicity condition reads

$$
\Omega \wedge \mathcal{F}=0 \quad \Leftrightarrow \quad \mathcal{F}^{0,2}=0
$$

and, second, applying the covariant differential to (4.3a), and using the Bianchi identity as well as (4.3a) yields

$$
\mathrm{d} \Omega \wedge \mathcal{F}=\left[\left(W_{1}^{+}+\mathrm{i} W_{1}^{-}\right) \omega \wedge \omega+\left(W_{2}^{+}+\mathrm{i} W_{2}^{-}\right) \wedge \omega\right] \wedge \mathcal{F}=0 .
$$

The last equation, although a mere consequence of (4.3a), depends strongly on the type of SU(3)-manifold under consideration. For example, on nearly Kähler manifolds one has

$$
\mathrm{d} \Omega \propto \omega \wedge \omega \stackrel{(4.3 \mathrm{~b})}{\Longrightarrow} \quad \omega \wedge \omega \wedge \mathcal{F}=0 \quad \Leftrightarrow \quad \omega\lrcorner \mathcal{F}=0,
$$


whereas on half-flat $\mathrm{SU}(3)$-manifolds this is not true as $\mathrm{d} \Omega \neq \kappa \omega \wedge \omega$. For Calabi-Yau spaces, on the other hand, (4.3b) is trivial as $\mathrm{d} \Omega=0$, and the condition $\omega\lrcorner \mathcal{F}=0$ is added as an additional stability condition for the holomorphic instanton bundle [29-31].

Following [64], one considers a complex vector bundle $\mathcal{V} \rightarrow M^{6}$ of rank $k$ on which we are given an instanton $\Gamma$ with curvature $R_{\Gamma}$. Here this vector bundle will be the tangent bundle of 6-manifolds arising as certain conical extensions of SU(2) 5-manifolds $M^{5}$, just as we considered in the previous section. We then generalize this instanton $\Gamma$ by extending it to a connection $\mathcal{A}$ with curvature $\mathcal{F}$ by the ansatz

$$
\mathcal{A}=\Gamma+X_{\mu} e^{\mu} \quad \text { and } \quad \mathcal{F}=\mathrm{d} \mathcal{A}+\mathcal{A} \wedge \mathcal{A},
$$

where $\mu=1, \ldots, 5$ and

$$
\Gamma=\Gamma^{i} \hat{I}_{i}, \quad i=6,7,8
$$

Here $\hat{I}_{i}$ is a representation of the $\mathrm{SU}(2)$-generators $I_{i}$ on the fibres $\mathbb{R}^{6}$ of the bundle, and $\Gamma^{i}$ are the components of an $\mathfrak{s u}(2)$-connection on the tangent bundle of $M^{6}$. Furthermore, $X_{\mu}$ are matrices from $\operatorname{End}\left(\mathbb{R}^{6}\right)$.

The computation of $\mathcal{F}$ with the ansatz for $\mathcal{A}$ yields

$$
\begin{aligned}
\mathcal{F}=R_{\Gamma}+\mathrm{d} X_{\mu} \wedge e^{\mu}+T_{6 \nu}^{\mu} X_{\mu} e^{6} \wedge e^{\nu}+\frac{1}{2}\left(\left[X_{\mu}, X_{\nu}\right]\right. & \left.+T_{\mu \nu}^{\sigma} X_{\sigma}\right) e^{\mu} \wedge e^{\nu} \\
& +\Gamma^{i}\left(\left[\hat{I}_{i}, X_{\mu}\right]-f_{i \mu}^{\nu} X_{\nu}\right) \wedge e^{\mu} .
\end{aligned}
$$

Herein, $T$ denotes the torsion of the connection $\Gamma$.

In order to simplify this further, we investigate the matrices $X_{\mu}$ and their transformation behavior under a change of $e$. By construction, $X_{\mu} e^{\mu}$ is the local representation of an $A d$-equivariant 1-form $X$ on the gauge principal bundle, which here coincides with the $\mathrm{SU}(3)$-subbundle $\mathcal{P}$ of the frame bundle of $M^{6}$ that constitutes the $\mathrm{SU}(3)$-structure. Note that, in the aforementioned cases, $\mathcal{P}$ contains a principal $\mathrm{SU}(2)$-subbundle $\mathcal{Q}$; the latter is the principal bundle for the connection $\Gamma$. Now let $e$ and $e^{\prime}$ be two local sections of $\mathcal{Q} \subset \mathcal{P}$ over some $U \subset M^{6}$ related by an $\mathrm{SU}(2)$-transformation $L: U \rightarrow S U(2)$. The components $X_{\mu}^{\prime}$ and $X_{\mu}$ of $X$ with respect to $e^{\prime}$ and $e$ are related via

$$
X_{\mu}^{\prime}=A d\left(L^{-1}\right) \circ X_{\nu} \rho(L)_{\mu}^{\nu} .
$$

Here $\rho$ is the representation of $\mathrm{SU}(2)$ on $\mathbb{R}^{5}$ which is the typical fiber of $T M^{5}$. It coincides with the representation $A d_{S U(3)}: \mathrm{SU}(2) \rightarrow \operatorname{End}(\mathfrak{m})$, where $\mathfrak{s u}(3)=\mathfrak{s u}(2) \oplus \mathfrak{m}$ and one has the identification $\mathfrak{m} \simeq T_{x} M^{5}$.

Since we will search for $\mathfrak{s u}(3)$-valued connections $\mathcal{A}$, we consider the $\mathfrak{s u}(3)$-generator algebra

$$
\begin{aligned}
{\left[\hat{I}_{i}, \hat{I}_{j}\right] } & =f_{i j}{ }^{k} \hat{I}_{k}, & i, j, k=6,7,8 \\
{\left[\hat{I}_{i}, \hat{I}_{\mu}\right] } & =f_{i \mu}{ }^{\nu} \hat{I}_{\nu}, & \mu, \nu=1,2,3,4,5 \\
{\left[\hat{I}_{\mu}, \hat{I}_{\nu}\right] } & =f_{\mu \nu}{ }^{i} \hat{I}_{i}+f_{\mu \nu}{ }^{\sigma} \hat{I}_{\sigma} ; . &
\end{aligned}
$$


The generators with indices $i, j, k$ belong to the $\mathfrak{s u}(2)$ subalgebra, and the indices $\mu, \nu, \sigma$ correspond to its orthogonal complement $\mathfrak{m}$ in the $\mathrm{SU}(2)$-invariant splitting

$$
\mathfrak{s u}(3)=\mathfrak{s u}(2) \oplus \mathfrak{m} .
$$

Generically, only $X$ is well-defined globally, rather than the component maps $X_{\mu}$. The latter strongly depend on the choice of the local frame $e$ and, therefore, we have no control over their behavior in general. That would be different, if the components $X_{\mu}$ were independent of the trivialization of the involved bundles, that is, if the $X_{\mu}$ were invariant under the aforementioned transformations (4.8) that change the local frames. Furthermore, since $\mathrm{SU}(2)$ is connected, this is equivalent to the infinitesimal version of the invariance, i.e.

$$
\left[\hat{I}_{i}, X_{\mu}\right]=\rho_{*}\left(I_{i}\right)_{\mu}^{\nu} X_{\nu}=f_{i \mu}{ }^{\nu} X_{\nu}
$$

Note that this simplification implies that the $X_{\mu}$ are independent of the choice of frame adapted to the $\mathrm{SU}(2)$-structure $\mathcal{Q}$; hence, we can choose them to vary with the cone direction only. Condition (4.11) appeared, for example, in [65, 66] on coset spaces, where equivariant connections have been constructed. We will in the following refer to (4.11) as the equivariance condition, despite its different origin in this context.

Inserting this simplification and the accompanying consistency condition (4.11) into (4.7), we are left with

$$
\mathcal{F}=R_{\Gamma}+\left(\dot{X}_{\mu}+T_{6 \mu}^{\nu} X_{\nu}\right) e^{6} \wedge e^{\mu}+\frac{1}{2}\left(\left[X_{\mu}, X_{\nu}\right]+T_{\mu \nu}^{\sigma} X_{\sigma}\right) e^{\mu} \wedge e^{\nu} .
$$

Here the dot denotes the derivation in the cone direction. In any case, the instanton condition is the requirement that the 2 -form part of $\mathcal{F}$ takes values in a certain subbundle of $\Lambda^{2} T^{*} M^{6}$, which we call the instanton bundle. Anticipating that 2-forms of the general form $e^{6} \wedge e^{\sigma}+\frac{1}{2} N_{\mu \nu}^{\sigma} e^{\mu} \wedge e^{\nu}$, with $N$ to be determined from the geometry under consideration, are local sections of this instanton bundle, we add a zero to the above expression and obtain

$$
\begin{aligned}
\mathcal{F}= & R_{\Gamma}+\left(\dot{X}_{\mu}+T_{6 \mu}^{\nu} X_{\nu}\right)\left(e^{6} \wedge e^{\mu}+\frac{1}{2} N_{\sigma \rho}^{\mu} e^{\sigma} \wedge e^{\rho}\right) \\
& +\frac{1}{2}\left(\left[X_{\mu}, X_{\nu}\right]+T_{\mu \nu}^{\sigma} X_{\sigma}-N_{\mu \nu}^{\sigma}\left(\dot{X}_{\sigma}+T_{6 \sigma}^{\rho} X_{\rho}\right)\right) e^{\mu} \wedge e^{\nu} .
\end{aligned}
$$

As argued above, $R_{\Gamma}$ and the second term already are instantons. Thus, we are left to require that the last term satisfies the instanton equation; this leads us to

$$
\left[X_{\mu}, X_{\nu}\right]+T_{\mu \nu}^{\sigma} X_{\sigma}=N_{\mu \nu}^{\sigma}\left(\dot{X}_{\sigma}+T_{6 \sigma}^{\rho} X_{\rho}\right)+\mathcal{N}_{\mu \nu}
$$

where $\mathcal{N}$ has to be an instanton on $M^{6}$ that compensates for the $\mathfrak{s u}(2)$-component of the left-hand-side commutator. Hence, $\mathcal{N}$ can only be a linear combination of the three instantons [22] $f_{\mu \nu}{ }^{i} e^{\mu} \wedge e^{\nu}$ for $i=6,7,8$, which depends on the cone coordinate. That is,

$$
\left[X_{\mu}, X_{\nu}\right]+T_{\mu \nu}^{\sigma} X_{\sigma}=N_{\mu \nu}^{\sigma}\left(\dot{X}_{\sigma}+T_{6 \sigma}^{\rho} X_{\rho}\right)+f_{\mu \nu}{ }^{i} \mathcal{N}_{i} .
$$

In summary, we are searching for $\mathfrak{m}$-valued matrices $X_{\mu}$ that solve equations (4.11) and (4.15), as these will give rise to new instantons on the considered manifolds. 


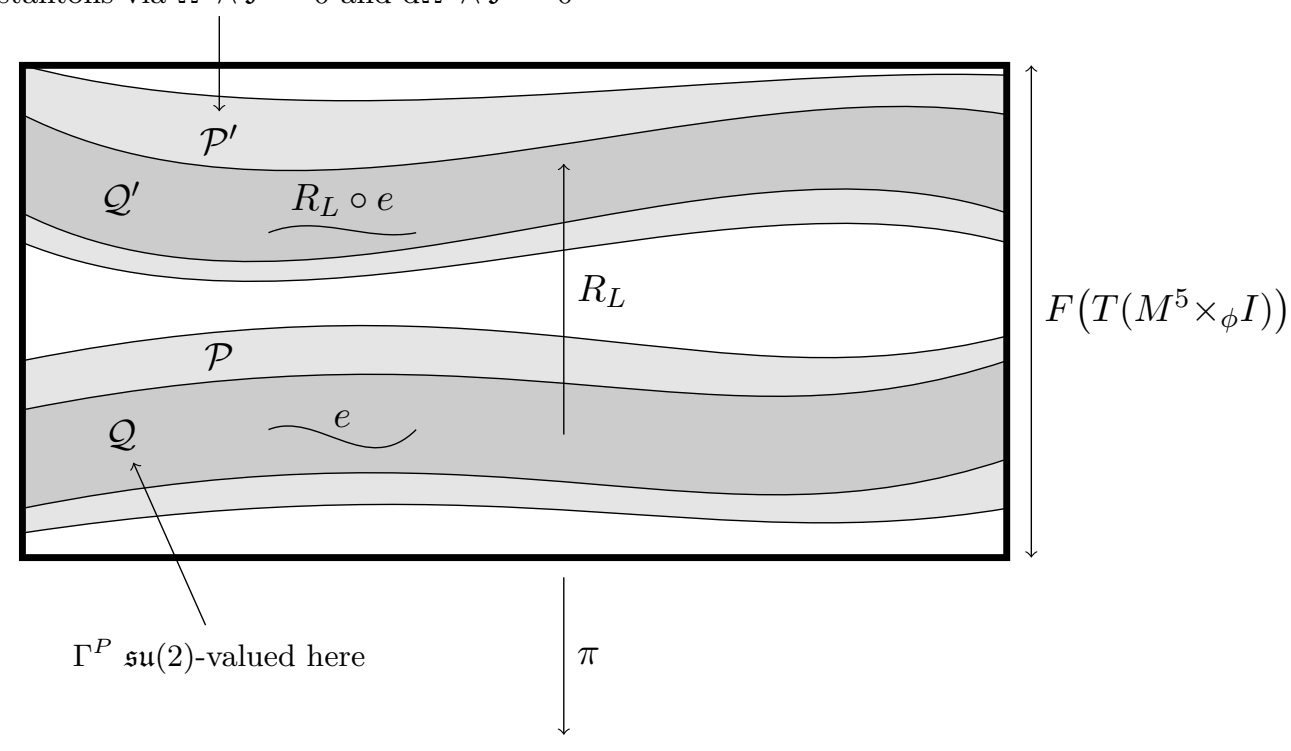

$M^{5} \times_{\phi} I$

Figure 1. A schematic depiction of the different principal bundles involved in the definition of the instanton condition: $\mathcal{Q}$ and $\mathcal{P}$ are the $\mathrm{SU}(2)$ - and $\mathrm{SU}(3)$-bundles, respectively, which originate from the Sasaki-Einstein structure on $M^{5}$. The transformation $L$ defines the principal bundles $\mathcal{Q}^{\prime}$ and $\mathcal{P}^{\prime}$, which again are $\mathrm{SU}(2)$ and $\mathrm{SU}(3)$-bundles, respectively. All bundles under consideration are understood as principal subbundles of the frame bundle $F\left(T\left(M^{5} \times_{\phi} I\right)\right)$.

\subsection{Remarks on the instanton equation}

Before proceeding with the particular cases of the nearly Kähler sine-cone and the half-flat cylinder, one needs to clarify an important point regarding the transformations of coframes mentioned in section 3 .

The $\mathrm{SU}(2)$-structure on the Sasaki-Einstein 5-manifold is understood as an $\mathrm{SU}(2)$ principal bundle $\mathcal{Q}$, a subbundle of the frame bundle $F\left(T M^{5}\right)$. The warped product $M^{5} \times_{\phi} I$ (cf. (3.2)) is equipped with an SU(3)-structure via (3.4) and the corresponding principal bundle is denoted with $\mathcal{P} \subset F\left(T\left(M^{5} \times_{\phi} I\right)\right.$ ) (cf. figure 1). However, $\mathcal{P}$ is not the $\mathrm{SU}(3)$-structure one is interested in, i.e. in our cases it is neither nearly Kähler nor halfflat. The constructions of subsections 3.3 and 3.4 rely on transformations of the coframes on $M^{5}$ : they generate a different $\mathrm{SU}(2)$-structure $\mathcal{Q}^{\prime}$ that can be extended to the desired $\mathrm{SU}(3)$-structure $\mathcal{P}^{\prime}$ on the warped product. An important observation is the following: for a $G$-structure $\mathcal{Q}$ the bundle $\mathcal{Q}^{\prime}$ defined via $\mathcal{Q}^{\prime}=R_{L} \mathcal{Q}$ is a $G$-structure if and only if $L$ is a map from the base to the normalizer $N_{\mathrm{GL}(6, \mathbb{R})}(G)$, cf. (3.3).

The crux of the instanton equation is the following: the defining forms $\left(\omega^{\prime}, \Omega^{\prime}\right)$ stem from $\mathcal{P}^{\prime}$, whereas the canonical connection $\Gamma^{P}$ belongs to $\mathcal{Q}$ and is trivially lifted to an instanton on $\mathcal{P}$. Let us denote by $e \in \Gamma(U, \mathcal{Q})$ an adapted frame for $\mathcal{Q}$. Then by construction $e^{\prime}=:\left(R_{L} \circ e\right) \in \Gamma\left(U, \mathcal{Q}^{\prime}\right)$ is an adapted frame for $\mathcal{Q}^{\prime}$. By standard results, the connection 
1-forms of $\mathcal{A}$ transform under a change of section as

$$
e^{\prime *} \mathcal{A}=\operatorname{Ad}\left(L^{-1}\right) \circ e^{*} \mathcal{A}+L^{-1} \mathrm{~d} L
$$

The employed extension $\mathcal{A}=\Gamma^{P}+X$ relies on the splitting (4.10) such that $X$ corresponds to $\mathfrak{m}$-valued 1-forms. However, this only holds in the frame $e$, due to the following: starting with $\Gamma^{P}$ on $\mathcal{Q}$, one has a purely $\mathfrak{s u}(2)$-valued connection. Applying any transformation $L$ to $\mathcal{Q}, \Gamma^{P}$ is generically not an $\mathfrak{s u}(2)$-valued connection on $\mathcal{Q}^{\prime}$. This is due to the fact that $L^{-1} \mathrm{~d} L$, in general, takes values in the Lie-algebra of $N_{\mathrm{GL}(6, \mathbb{R})}(\mathrm{SU}(2))$ instead of $\mathfrak{s u}(2)$. Therefore, one cannot simply take $e^{\prime *} \Gamma^{P}$ as an starting point for some ansatz like $e^{\prime *} \mathcal{A}=$ $e^{\prime *} \Gamma^{P}+X^{\prime}{ }_{\mu} e^{\prime \mu}$.

For the cases under consideration, $L$ depends (at most) on the cone direction $r$. Hence, one has that $\operatorname{Ad}\left(L^{-1}\right) \circ e^{*} \mathcal{A}$ is $\mathfrak{s u}(2)$-valued and $L^{-1} \mathrm{~d} L \propto \mathrm{d} r$, but generically not $\mathfrak{s u}(2)$ valued. The immediate consequences are the following:

- For instance, on the nearly Kähler sine-cone one has to perform all calculations in the frame $e$, because for the derivation of subsection 4.1 we employed a section of the bundle on which $\Gamma^{P}$ is an $\mathfrak{s u}(2)$-valued connection. We will, however, compute $e^{\prime *} \Gamma^{P}$ explicitly in subsection 4.3 .2 and demonstrate that it yields an $\mathfrak{s u}(3)$-valued instanton on the sine-cone.

- In contrast, the transformation for the half-flat cylinder (3.26) is, although a 2parameter family, base-point independent. Therefore, one is allowed to consider the frames $e$ as well as $e^{\prime}$ for this instanton equation, as $e^{*} \Gamma^{P}$ and $e^{\prime *} \Gamma^{P}$ are $\mathfrak{s u}(2)$ valued connection 1-forms. However, this raises the question whether the two extensions $X_{\mu} e^{\mu}$ and $X^{\prime}{ }_{\mu} e^{\prime \mu}$ are in any sense comparable. Unfortunately, the coframetransformations are only required to be $N_{\mathrm{GL}(6, \mathbb{R})}(\mathrm{SU}(2))$-valued, which implies that the $\mathfrak{m}$-piece will, in general, not be mapped into $\mathfrak{m}$ or even $\mathfrak{s u}(3)$. Hence, one cannot simply compare both extensions, but it is admissible to consider both cases.

In summary, these remarks were not relevant for the cases studied for example in $[22,64]$ or our earlier results [53], because the construction of the $G$-structures on the warped product $M^{5} \times{ }_{\phi} I$ followed immediately from the chosen frame on $M^{5}$. In other words, no (base-point dependent) transformation of coframes was necessary. Even on our KT-and HKT-sine cones of [53], the relevant rescaling (3.15) does not affect the computations due to conformal equivalence to the cylinder. However, here the situation is more involved and a careful analysis is mandatory.

\subsection{Instantons on nearly Kähler sine-cones}

\subsubsection{Matrix equations — Part I}

The set-up for the nearly Kähler sine-cone has been described in section 3.3. In particular, we are investigating extensions of the connection $\Gamma^{P}$ on the sine-cone in this subsection. $M^{6}$ being a nearly Kähler manifold, the instanton equation with respect to the coframe $e^{\mu}$ 
is equivalent to

$$
\begin{aligned}
\omega \wedge \omega \wedge \mathcal{F}=0 & \Leftrightarrow & \omega^{\hat{\mu} \hat{\nu}} \mathcal{F}_{\hat{\mu} \hat{\nu}}=0, \\
\Omega \wedge \mathcal{F}=0 & \Leftrightarrow & \Omega^{\hat{\sigma} \hat{\mu} \hat{\nu}} \mathcal{F}_{\hat{\mu} \hat{\nu}}=0 \quad \text { for } \quad \hat{\sigma}=1, \ldots, 6 .
\end{aligned}
$$

The seven equations (4.17) restrict the space of admissible 2-forms, and the instanton bundle, which is locally isomorphic ${ }^{3}$ to the subspace $\mathfrak{m}$, is spanned by

$$
\begin{aligned}
& e^{5} \wedge e^{6}-\frac{\Lambda \sin \varphi}{4}\left(\sin \varphi \eta_{a b}^{1}+\cos \varphi \eta_{a b}^{3}\right) e^{a} \wedge e^{b} \text { and } \\
& e^{a} \wedge e^{6}-\Lambda \sin \varphi\left(\sin \varphi \eta_{b}^{1 a}+\cos \varphi \eta_{b}^{3 a}\right) e^{b} \wedge e^{5} .
\end{aligned}
$$

This can be seen either by direct computation or by the explicit form of the projectors from $\mathfrak{s o}(6)$ to $\mathfrak{s u}(3)$ of [51]. Here we have used the Riemannian metric to pull up one of the indices of $\eta^{3}$, and from here on we use $e^{6}=\mathrm{d} r$.

A 6-dimensional representation of $\mathfrak{m}$ can be chosen as in [22, 64],

$$
\begin{array}{rlrl}
\left(\hat{I}_{5}\right)_{a}^{b} & =\frac{1}{2} \eta_{a b}^{3}, & -\left(\hat{I}_{5}\right)_{5}^{6}=\left(\hat{I}_{5}\right)_{6}^{5}=1, \\
-\left(\hat{I}_{a}\right)_{b}^{6} & =\left(\hat{I}_{a}\right)_{6}^{b}=\delta_{a}^{b}, \quad\left(\hat{I}_{a}\right)_{b}^{5}=-\left(\hat{I}_{a}\right)_{5}^{b}=\eta_{a b}^{3},
\end{array}
$$

from which one obtains the structure constants

$$
f_{5 a}{ }^{b}=\frac{3}{2} \eta_{a}^{3}{ }_{a}^{b} \quad \text { and } \quad f_{a b}{ }^{5}=2 \eta_{a b}^{3} .
$$

The torsion components of the canonical $\mathfrak{s u}(2)$-connection $\Gamma^{P}$ in the unrotated frame $e^{\mu}$ read

$$
\begin{aligned}
& T_{a b}^{5}=-2 \eta_{a b}^{3}=-f_{a b}{ }^{5}, \\
& T_{b 5}^{a}=-\frac{3}{2}\left(\eta^{3}\right)^{a}{ }_{b}=-f_{b 5}{ }^{a} .
\end{aligned}
$$

With the chosen representation and by inserting the ansatz

$$
\mathcal{A}=\Gamma^{P}+X_{\mu} e^{\mu}
$$

into (4.17), one obtains the non-vanishing components $N_{\mu \nu}^{\rho}$ of the parametrization (4.15) as follows:

$$
N_{a b}^{5}=\frac{\Lambda \sin \varphi}{2}\left(\sin \varphi \eta_{a b}^{1}+\cos \varphi \eta_{a b}^{3}\right) \quad \text { and } \quad N_{b 5}^{a}=\Lambda \sin \varphi\left(\sin \varphi \eta_{b}^{1 a}+\cos \varphi \eta_{b}^{3 a}\right)
$$

Finally, the matrix equations for $X_{\mu}$ read

$$
\begin{aligned}
{\left[\hat{I}_{i}, X_{\mu}\right] } & =f_{i \mu}{ }^{\nu} X_{\nu} \\
{\left[X_{a}, X_{b}\right] } & =\frac{\Lambda \sin \varphi}{2}\left(\sin \varphi \eta_{a b}^{1}+\cos \varphi \eta_{a b}^{3}\right) \dot{X}_{5}+2 \eta_{a b}^{3} X_{5}+f_{a b}{ }^{i} \mathcal{N}_{i}, \\
{\left[X_{5}, X_{a}\right] } & =\Lambda \sin \varphi\left(\sin \varphi \eta_{a}^{1}{ }_{a}^{b}+\cos \varphi \eta_{a}^{3}{ }_{a}^{b}\right) \dot{X}_{b}+\frac{3}{2} \eta_{a}^{3}{ }_{a} X_{b}
\end{aligned}
$$

\footnotetext{
${ }^{3}$ One employs the identification $\mathfrak{s o}(6) \simeq \Lambda^{2}\left(\mathbb{R}^{6}\right)$ to obtain 2 -forms from antisymmetric $6 \times 6$-matrices.
} 
where the first line is just the equivariance condition (4.11). The dot-notation means $\dot{Y} \equiv \frac{\mathrm{d}}{\mathrm{d} r} Y$. An obvious solution to (4.24) is $X_{\mu} \equiv 0$, which yields the instanton solution $\mathcal{A}=\Gamma^{P}$ that is the lift of the instanton $\Gamma^{P}$ from $M^{5}$ to the sine-cone $C_{s}\left(M^{5}\right)$.

Consider the ansatz

$$
X_{a}=\psi(r)\left(\exp \left(\xi \eta^{3}\right)\right)_{a}^{b} \hat{I}_{b}, \quad \text { for } \quad \xi \in[0,2 \pi) \quad \text { and } \quad X_{5}=\chi(r) \hat{I}_{5},
$$

which respects equivariance due to $\left[\eta^{\alpha}, \bar{\eta}^{\beta}\right]=0$. Here, $\xi$ is a parameter, and $\psi(r), \chi(r)$ are two functions depending only on the cone direction $r$. Inserting (4.25) into (4.24) yields

$$
\mathcal{N}_{i}=\psi^{2}(r) \hat{I}_{i} \quad \text { for } \quad i=6,7,8
$$

as well as the following differential equations

$$
\frac{\Lambda}{2} \dot{\chi}(r) \sin (2 \varphi)=4\left(\psi^{2}(r)-\chi(r)\right) \quad \text { and } \quad \frac{\Lambda}{2} \dot{\psi}(r) \sin (2 \varphi)=\frac{3}{2} \psi(r)(\chi(r)-1),
$$

which are subject to the constraints

$$
\frac{\Lambda}{2} \dot{\psi}(r) \sin ^{2} \varphi=\frac{\Lambda}{2} \dot{\chi}(r) \sin ^{2} \varphi=0 .
$$

As a matter of fact, these equations (4.27) hold for any value of $\xi \in[0,2 \pi)$. The solutions to (4.27) are readily obtained to be the following:

- $(\psi, \chi)=(\mathbf{0}, \mathbf{0})$ : this is, of course, the trivial solution of $(4.24)$, but is still required for consistency as it confirms that $\Gamma^{P}$ satisfies the $\Omega_{s}$-instanton condition on $M^{6}$.

- $(\psi, \chi)=(\mathbf{1}, \mathbf{1})$ : here we obtain an extension of the original instanton $\Gamma^{P}$. Despite being an $\Omega_{s}$-instanton, this newly obtain instanton is a mere lift of an instanton in $M^{5}$ as it does not have any dependence on the cone direction.

- $(\psi, \chi)=(-\mathbf{1}, \mathbf{1})$ : again, we obtain an extension which is, however, a lift of an $M^{5}$-instanton. Note that the existence of this solutions follows from $\xi \mapsto \xi+\pi$, as $\left(\exp \left(\pi \eta^{3}\right)\right)_{a}^{b}=-\delta_{a}{ }^{b}$.

Hence, we have a one-parameter family of $\mathfrak{s u}(3)$-valued instantons given by

$$
A=\Gamma^{P}+\left(\exp \left(\xi \eta^{3}\right)\right)_{a}^{b} \hat{I}_{b} \otimes e^{a}+\hat{I}_{5} \otimes e^{5} .
$$

To summarize, the ansatz solving the matrix equations (4.24) generates isolated instanton solutions which can all be interpreted as lifts of connections living on $M^{5}$. The nontrivial solutions are $\mathfrak{s u}(3)$-valued connections; whereas the trivial solution is a purely $\mathfrak{s u}(2)$ valued connection.

Remarks: first, the family solutions (4.28) can be seen to be gauge orbit if we recall that $\left(\eta^{3}\right)_{\mu}^{\nu} \propto f_{5 \mu}{ }^{\nu}=\operatorname{ad}\left(I_{5}\right)_{\mu}^{\nu}$ and then $\exp \left(\xi \eta^{3}\right) \propto \operatorname{Ad}\left(\exp \left(I_{5}\right)\right)$. Nevertheless, this gauge symmetry clarifies the origin of the $\psi$-reflection symmetry of the solutions. 
Second, in the same manner as in our previous studies [53] we can equivalently provide the matrix equations on the conformally equivalent cylinder with coordinate $\tau$ as follows:

$$
\begin{aligned}
{\left[\hat{I}_{i}, X_{\mu}\right] } & =f_{i \mu}^{\nu} X_{\nu} \\
{\left[X_{a}, X_{b}\right] } & =\frac{1}{2}\left(\sin \varphi \eta_{a b}^{1}+\cos \varphi \eta_{a b}^{3}\right) \frac{\mathrm{d}}{\mathrm{d} \tau} X_{5}+2 \eta_{a b}^{3} X_{5}+f_{a b}{ }^{i} \mathcal{N}_{i}, \\
{\left[X_{5}, X_{a}\right] } & =\left(\sin \varphi \eta_{a}^{1}{ }_{a}^{b}+\cos \varphi \eta_{a}^{3}{ }_{a}^{b}\right) \frac{\mathrm{d}}{\mathrm{d} \tau} X_{b}+\frac{3}{2} \eta^{3}{ }_{a}^{b} X_{b} .
\end{aligned}
$$

Further, the limit $\Lambda \rightarrow \infty$ (with $\varphi=\frac{r}{\Lambda} \rightarrow 0$ and keeping $r$ fixed) transforms the sinecone into the Calabi-Yau cone, as mentioned in subsection 3.3. In this limit, the matrix equations (4.29) take the following form:

$$
\left[X_{a}, X_{b}\right]=f_{a b}{ }^{5}\left(X_{5}+\frac{1}{4} \dot{X}_{5}\right)+f_{a b}{ }^{i} \mathcal{N}_{i} \quad \text { and } \quad\left[X_{5}, X_{a}\right]=f_{5 a}{ }^{b}\left(X_{b}+\frac{2}{3} \dot{X}_{b}\right)
$$

which are exactly the same equations as on the Kähler-torsion sine-cone of our early results [53]. Applying the $\tau$-dependent version of the ansatz (4.25) yields

$$
\dot{\chi}(\tau)=4\left(\psi^{2}(\tau)-\chi(\tau)\right) \quad \text { and } \quad \dot{\psi}(\tau)=\frac{3}{2} \psi(\tau)(\chi(\tau)-1) .
$$

Obviously, all constant solutions found above are still instantons on the CY-cone, but the reduced equations do not automatically enforce constant $\chi$ and $\psi$. Finally, note that (4.31) is, of course, equivalent to (4.27) in the limit $\Lambda \rightarrow \infty$ as the constraint on the derivatives vanishes.

Third, the sine-cone is a conifold with two conical singularities, here at $\varphi=0$ and $\varphi=\pi$. One observes that the coefficient functions, i.e. $\cos \varphi$ and $\sin \varphi$, of (4.24) as well as our solutions are well-behaved at the singular points. However, recall the remark from subsection 3.3 that the defining sections of the $\mathrm{SU}(3)$-structure become trivial at these singular points; hence, the instanton condition is not well-defined there. Yet, in principal one could continue the gauge field to these points.

\subsubsection{Nearly Kähler canonical connection}

In this section we construct the canonical $\mathfrak{s u}(3)$-connection of the nearly Kähler sine-cone. It turns out that we obtain an instanton for the $\mathrm{SU}(3)$-structure that is not the lift of an instanton on $M^{5}$; furthermore, this instanton is of the form (4.22) presented above. On the 5-manifold $M^{5}$ the Maurer-Cartan equations read

$$
\begin{aligned}
\mathrm{d} e^{a} & =-\left(\Gamma^{P}\right)_{b}^{a} \wedge e^{b}+\frac{1}{2} T_{\mu \nu}^{a} e^{\mu} \wedge e^{\nu}, \\
\mathrm{d} e^{5} & =-\left(\Gamma^{P}\right)_{5}^{5} \wedge e^{5}+\frac{1}{2} T_{\mu \nu}^{5} e^{\mu} \wedge e^{\nu},
\end{aligned}
$$

where the torsion components are given by (cf. $[22,64])$

$$
T_{b 5}^{a}=-\frac{3}{2} \eta_{b}^{3 a} \quad \text { and } \quad T_{a b}^{5}=-2 \eta_{a b}^{3} .
$$


In particular, the last identity implies $\left(\Gamma^{P}\right)_{5}^{5}=0$ due to the Sasaki-Einstein relation $\mathrm{d} e^{5}=-2 \omega^{3}$.

Next, we are interested in the Maurer-Cartan equations for the frame $e_{s}^{\mu}$ resulting from the rotation (3.21) and rescaling (3.23) of the $\mathrm{SU}(2)$-structure. With respect to coframes $e$ adapted to $\mathcal{Q}$, the canonical $\mathfrak{s u}(2)$-connection $\Gamma^{P}$ has components

$$
\left(\Gamma^{P}\right)_{\mu}^{\nu}=\left(\Gamma^{P}\right)^{i} f_{i \mu}{ }^{\nu} \quad \text { with } \quad\left(f_{i a}{ }^{b}\right) \propto \bar{\eta}^{\alpha(i)},
$$

where $\alpha(i)=i-5$ and $\bar{\eta}^{\alpha}$ are the anti-self-dual 't Hooft tensors. Noting that $\left[\eta^{\alpha}, \bar{\eta}^{\beta}\right]=0$ for all $\alpha, \beta$, we see that the components of the canonical $\mathfrak{s u}(2)$-connection are unaffected by the homogeneous part of the transformation (4.16) with

$$
L(r)=\Lambda \sin (\varphi)\left(\begin{array}{cc}
\exp \left(\frac{\varphi}{2} \eta^{2}\right)_{4 \times 4} & 0_{4 \times 2} \\
0_{2 \times 4} & \mathbb{1}_{2 \times 2}
\end{array}\right) \in N_{\mathrm{GL}(6, \mathbb{R})}(\mathrm{SU}(2)),
$$

which realizes the rotation (3.21) and the rescaling (3.23). In detail, the transformation reads $\left(\Gamma^{P}\right)_{b}^{a}=L_{c}^{a}\left(\Gamma^{P}\right)_{d}^{c}\left(L^{-1}\right)_{b}^{d}$. A straightforward computation yields

$$
\begin{aligned}
\mathrm{d} e_{s}^{a}=-\left(\Gamma^{P}\right)_{b}^{a} \wedge e_{s}^{b} & -\frac{\cot \varphi}{\Lambda}\left(e_{s}^{a} \wedge e_{s}^{6}+\eta^{3 a}{ }_{b} e_{s}^{b} \wedge e_{s}^{5}\right)-\frac{\cot \varphi}{2 \Lambda} \eta_{b}^{3 a} e_{s}^{b} \wedge e_{s}^{5} \\
& -\frac{1}{2 \Lambda}\left(\eta^{2 a}{ }_{b} e_{s}^{b} \wedge e_{s}^{6}-\eta^{1 a}{ }_{b} e_{s}^{b} \wedge e_{s}^{5}\right)+\frac{1}{\Lambda} \eta^{1 a}{ }_{b} e_{s}^{b} \wedge e_{s}^{5}, \\
\mathrm{~d} e_{s}^{5}= & -\frac{\cot \varphi}{\Lambda}\left(e_{s}^{5} \wedge e_{s}^{6}+\eta_{a b}^{3} e_{s}^{a} \wedge e_{s}^{b}\right)+\frac{1}{\Lambda} \eta_{a b}^{1} e_{s}^{a} \wedge e_{s}^{b}, \\
\mathrm{~d} e_{s}^{6}= & 0 .
\end{aligned}
$$

It is important to realize that, although the components $\left(\Gamma^{P}\right)_{b}^{a}$ used in (4.36) coincide with the components of the lift of the canonical connection on the Sasaki-Einstein 5-manifold to the cylinder, the transformed coframe $e_{s}^{\mu}$ is used since we are on the nearly Kähler sinecone. Thus, $\left(\Gamma^{P}\right)_{b}^{a}$ no longer comprises the canonical $\mathfrak{s u}(2)$-connection; however, it forms a different $\mathfrak{s u}(2)$-valued connection $\Gamma_{\mathfrak{s u}(2)}$. This is because the inhomogeneous term in (4.16), which results from the change of basis, has been split off.

Introducing an almost complex structure $J$ via demanding

$$
\Theta_{s}^{1}=e_{s}^{1}+\mathrm{i} e_{s}^{2}, \quad \Theta_{s}^{2}=e_{s}^{3}+\mathrm{i} e_{s}^{4}, \quad \Theta_{s}^{3}=\mathrm{i}\left(e_{s}^{5}+\mathrm{i} e_{s}^{6}\right)
$$

to be $(1,0)$-forms yields

$$
\mathrm{d}\left(\begin{array}{c}
\Theta_{s}^{1} \\
\Theta_{s}^{2} \\
\Theta_{s}^{3}
\end{array}\right)=-\underbrace{\left(\begin{array}{ccc}
\hat{\Gamma}_{\mathfrak{s u}(2) 1}+\frac{\mathrm{i} \cot \varphi}{2 \Lambda} e_{s}^{5} & \hat{\Gamma}_{\mathfrak{s u}(2) 2}^{1} & -\frac{\cot \varphi}{\Lambda} \Theta_{s}^{1}-\frac{1}{2 \Lambda} \Theta_{s}^{\overline{2}} \\
\hat{\Gamma}_{\mathfrak{s u}(2) 1}^{2} & \hat{\Gamma}_{\mathfrak{s u}(2) 2}^{2}+\frac{\mathrm{i} \cot \varphi}{2 \Lambda} e_{s}^{5} & -\frac{\cot \varphi}{\Lambda} \Theta_{s}^{2}+\frac{1}{2 \Lambda} \Theta_{s}^{\overline{1}} \\
\frac{\cot \varphi}{\Lambda} \Theta_{s}^{\overline{1}}+\frac{1}{2 \Lambda} \Theta_{s}^{2} & \frac{\cot \varphi}{\Lambda} \Theta_{s}^{\overline{2}}-\frac{1}{2 \Lambda} \Theta_{s}^{1} & -\frac{\mathrm{i} \cot \varphi}{\Lambda} e_{s}^{5}
\end{array}\right)}_{\text {canonical } \mathfrak{s u}(3) \text {-connection } \hat{\Gamma}_{\mathfrak{s u}(3)} \text { on sine-cone }} \wedge\left(\begin{array}{c}
\Theta_{s}^{1} \\
\Theta_{s}^{2} \\
\Theta_{s}^{3}
\end{array}\right) \underbrace{-\frac{1}{\Lambda}\left(\begin{array}{c}
\Theta_{s}^{\overline{2} \overline{3}} \\
\Theta_{s}^{3 \overline{1}} \\
\Theta_{s}^{\overline{1}} \overline{2}
\end{array}\right)}_{\text {NK-torsion } \hat{T}} .
$$

Here we used the shorthand notation $\Theta^{\bar{\alpha} \bar{\beta}} \equiv \Theta^{\bar{\alpha}} \wedge \Theta^{\bar{\beta}}$.

The connection 1-forms $\hat{\Gamma}_{\mathfrak{s u}(2) \alpha}^{\beta}$ with $\alpha, \beta=1,2$ are defined via the components $\left(\Gamma^{P}\right)_{a}^{b}$ by employing (4.32) and (4.36) as well as the change to the complex basis (4.37). We 
use the hat to indicate that we are considering the connection forms with respect to the complex basis $\Theta_{s}$ rather than the real basis $e_{s}$. Thus, the corresponding Maurer-Cartan equations read

$$
\mathrm{d} \Theta_{s}^{\alpha}=-\hat{\Gamma}_{\mathfrak{s u}(3))}^{\alpha} \wedge \Theta_{s}^{\beta}+\hat{T}^{\alpha} \quad \text { and } \quad \mathrm{d} \Theta_{s}^{\bar{\alpha}}=-\hat{\Gamma}_{\mathfrak{s u}(3)} \frac{\bar{\alpha}}{\bar{\beta}} \wedge \Theta_{s}^{\bar{\beta}}+\hat{T}^{\bar{\alpha}}
$$

Note that $\Gamma_{\mathfrak{s u}(3)}=\operatorname{diag}\left(\hat{\Gamma}_{\mathfrak{s u}(3)}, \hat{\Gamma}_{\mathfrak{s u}(3)}^{*}\right)$ is indeed a connection on $T M^{6}$, which can be seen from (4.39) and the fact that $\hat{T}$ transforms as a tensor. Furthermore, $\Gamma_{\mathfrak{s u}(3)}$ is an instanton because it satisfies the conditions of proposition 3.1 of [22].

The above result (4.38) can be brought into a more suggestive form by rewriting it as

$$
\begin{aligned}
\hat{\Gamma}_{\mathfrak{s u}(3)}= & \hat{\Gamma}_{\mathfrak{s u}(2)}+\frac{1}{2 \Lambda}\left(\begin{array}{ccc}
0 & 0 & -2 \cot \varphi \\
0 & 0 & 1 \\
2 \cot \varphi & -1 & 0
\end{array}\right) e_{s}^{1}+\frac{\mathrm{i}}{2 \Lambda}\left(\begin{array}{ccc}
0 & 0 & -2 \cot \varphi \\
0 & 0 & -1 \\
-2 \cot \varphi & -1 & 0
\end{array}\right) e_{s}^{2} \\
& +\frac{1}{2 \Lambda}\left(\begin{array}{ccc}
0 & 0 & -1 \\
0 & 0 & -2 \cot \varphi \\
1 & 2 \cot \varphi & 0
\end{array}\right) e_{s}^{3}+\frac{\mathrm{i}}{2 \Lambda}\left(\begin{array}{ccc}
0 & 0 & 1 \\
0 & 0 & -2 \cot \varphi \\
1-2 \cot \varphi & 0
\end{array}\right) e_{s}^{4} \\
& +\frac{\mathrm{i}}{2 \Lambda}\left(\begin{array}{ccc}
\cot \varphi & 0 & 0 \\
0 & \cot \varphi & 0 \\
0 & 0 & -2 \cot \varphi
\end{array}\right) e_{s}^{5} \\
= & \hat{\Gamma}_{\mathfrak{s u}(2)}+B_{\mu} \otimes e_{s}^{\mu},
\end{aligned}
$$

which reflects exactly the $X_{\mu}$-ansatz from (4.22). One can check that the matrices $B_{\mu}$ satisfy the equivariance condition (4.11). Thus, as $\Gamma_{\mathfrak{s u}(3)}$ is a connection on $T M^{6}$, one can infer by the same arguments as in section 4.1 that $\Gamma_{\mathfrak{s u}(2)}$ is a well-defined connection on $T M^{6}$. An alternative way to see that is to check that the inhomogeneous part, which has been split off in the transformation law (4.16) for the components of $\Gamma^{P}$, glues to globally well-defined 1-forms with values in the adjoint bundle of $\mathcal{P}$. This, however, holds due to the fact that the transformation $L$ given in (4.35) commutes with the $\mathrm{SU}(2)$ subgroup of $\mathrm{GL}(6, \mathbb{R})$, i.e. takes values in centralizer $C_{\mathrm{GL}(6, \mathbb{R})}(\mathrm{SU}(2))$.

Note that in the limit $\Lambda \rightarrow \infty$ (i.e. $\varphi=\frac{r}{\Lambda} \rightarrow 0$ ) the torsion on $C\left(M^{5}\right)$ vanishes, and $\hat{\Gamma}_{\mathfrak{s u}(3)}$ coincides with the connection corresponding to the $\chi=\psi=1$ case of [22], which has been stated to be the Levi-Civita connection of the cone. Furthermore, this is consistent with the observation that as $\hat{\Gamma}_{\mathfrak{s u}(3)}$ preserves the metric and as in the above limit its torsion vanishes, $\hat{\Gamma}_{\mathfrak{s u}(3)}$ has to converges to the Levi-Civita connection of the CY-cone.

\subsubsection{Matrix equations - Part II}

As pointed out above, there are two different $\mathfrak{s u}(2)$-valued connections on the nearly Kähler sine-cone. On the one hand, there is the lift of the canonical connection $\Gamma^{P}$ of the SasakiEinstein 5-manifold; on the other hand, there is $\Gamma_{\mathfrak{s u}(2)}$. Remarkably, the respective curvature 2 -forms coincide, i.e.

$$
R_{\Gamma^{P}}=R_{\Gamma_{\mathfrak{s u}(2)}}
$$


This stems from the fact that the generators of the two transformations (3.21) and (3.23), which lead from the cylinder to the sine-cone, commute with $\mathfrak{s u}(2)$. In other words, the inhomogeneous part of (4.16) yields an abelian flat part proportional to $e_{s}^{6}$. As a consequence, $\Gamma_{\mathfrak{s u}(2)}$ is another $\mathfrak{s u}(2)$-valued instanton on the sine-cone, since $\Gamma^{P}$ is an instanton itself. ${ }^{4}$ Therefore, we can use $\Gamma_{\mathfrak{s u}(2)}$ in the procedure described in section 4.1: one extends $\Gamma_{\mathfrak{s u}(2)}$ by some suitable 1-form $X_{\mu} e_{s}^{\mu}$ and investigates the conditions on $X_{\mu}$ such that the new connection is an instanton on the sine-cone.

However, we have to adjust the equations (4.24) due to the different torsion of $\Gamma_{\mathfrak{s u}(2)}$. Denoting by $T$ the torsion of $\Gamma^{P}$, the torsion of $\Gamma_{\mathfrak{s u}(2)}$ reads

$$
T_{\mathfrak{s u}(2)}^{\hat{\mu}}=T^{\hat{\mu}}+\frac{1}{\Lambda}\left(\delta_{\hat{\nu}}^{\hat{\mu}} \cot \varphi+\frac{1}{2} \eta_{\hat{\nu}}^{2 \hat{\mu}}\right) e_{s}^{6} \wedge e_{s}^{\hat{\nu}}
$$

where we defined $\eta_{\hat{\mu} \hat{\nu}}^{2}=\eta_{a b}^{2}$ for $\hat{\mu}, \hat{\nu}=a, b \in\{1, \ldots, 4\}$ and $\eta_{\hat{\mu} \hat{\nu}}^{2}=0$ whenever $\hat{\mu} \geq 5$ or $\hat{\nu} \geq 5$. The components of $N$ are the same as in subsection 4.3 .1 and, by inserting everything into (4.13), we obtain the matrix equations

$$
\begin{aligned}
{\left[\hat{I}_{i}, X_{\mu}\right] } & =f_{i \mu}{ }^{\nu} X_{\nu} \\
{\left[X_{a}, X_{b}\right] } & =\frac{1}{2} \eta_{a b}^{3} \dot{X}_{5}+\frac{1}{2 \Lambda}\left(5 \cot \varphi \eta_{a b}^{3}-4 \eta_{a b}^{1}\right) X_{5}+f_{a b}{ }^{i} \mathcal{N}_{i}, \\
{\left[X_{5}, X_{a}\right] } & =\eta^{3{ }^{b} \dot{X}_{b}}+\frac{1}{2 \Lambda}\left(5 \cot \varphi \eta_{a}^{3 b}-3 \eta_{a}^{1}{ }_{a}^{b}-\eta_{a}^{3}{ }^{c} \eta^{2}{ }_{c}{ }^{b}\right) X_{b},
\end{aligned}
$$

with the notation $\dot{Y}=\frac{\mathrm{d}}{\mathrm{d} r} Y$. Next, we use the matrices in (4.40) for the extension of $\Gamma_{\mathfrak{s u}(2)}$. Recall that we had defined auxiliary matrices $B_{\mu}$ that solve the equivariance condition (4.11) by writing (4.40) in the form

$$
\hat{\Gamma}_{\mathfrak{s u}(3)}=\hat{\Gamma}_{\mathfrak{s u}(2)}+B_{\mu} e_{s}^{\mu}
$$

and that the $B_{\mu}$ explicitly depend on $\varphi=\frac{r}{\Lambda}$. Hence, we may set

$$
X_{a}:=\psi(r) B_{a} \quad \text { and } \quad X_{5}:=\chi(r) B_{5}
$$

as in the usual procedure. ${ }^{5}$ The equivariance condition enforces the same coefficient function $\psi(r)$ for all four $B_{a}$. Inserting this $X_{\mu}$-ansatz in the matrix equations (4.43), one can first of all read off

$$
\mathcal{N}_{i}=\psi(r)^{2} \frac{1+4 \cot ^{2} \varphi}{4 \Lambda^{2}} I_{i}, \quad \text { for } \quad i=6,7,8,
$$

which is compatible with the assumptions on $\mathcal{N}$ used in subsection 4.1. Using this explicit form, we obtain the algebraic equation

$$
\psi(r)^{2}-\chi(r)=0
$$

\footnotetext{
${ }^{4}$ Recall subsection $4.2, \Gamma^{P}$ is a connection on the $\mathrm{SU}(2)$-bundle $\mathcal{Q}$, whereas $\Gamma_{\mathfrak{s u}(2)}$ is a connection on the SU(2)-bundle $\mathcal{Q}^{\prime}$.

${ }^{5}$ Note that in $(4.40)$ we have $B_{\mu} \in \operatorname{End}\left(\mathbb{C}^{3}\right)$. Here we used the identification $\mathbb{C} \simeq \mathbb{R}^{2}$ to obtain $B_{\mu} \in$ $\operatorname{End}\left(\mathbb{R}^{6}\right)$, which are necessary for the ansatz $(4.5)$.
} 
This then reduces the remaining equations to

$$
\dot{\chi}(r)=\dot{\psi}(r)=0 \quad \text { and } \quad \psi(r)(\chi(r)-1)=0 .
$$

Let us now comment on the three solutions to this system:

- $(\psi, \chi)=(\mathbf{0}, \mathbf{0})$ : to start with, there is the obvious trivial solution of (4.43). This is required for consistency, since $\Gamma_{\mathfrak{s u}(2)}$ is an instanton.

- $(\psi, \chi)=(\mathbf{1}, \mathbf{1})$ : this second solution is very important because it reproduces $\Gamma_{\mathfrak{s u}(3)}$ from subsection 4.3.2. We already knew from proposition 3.1 of [22] that this particular connection is an instanton on the nearly Kähler sine-cone, but here we confirmed it directly, using techniques completely different than those employed in [22]. In addition, this provides us with another way of constructing the canonical connection of the nearly Kähler sine-cone than the one we followed in subsection 4.3.2, namely as the extension of an $\mathfrak{s u}(2)$-valued instanton.

- $(\psi, \chi)=(-\mathbf{1}, \mathbf{1}):$ third, there is again the solution which results from the invariance of (4.43) under the simultaneous sign-flip $X_{a} \mapsto-X_{a}$ for $a=1,2,3,4$. Nevertheless, this solution is an additional instanton.

In summary, the solutions we obtained here are isolated $\mathfrak{s u}(3)$ - and $\mathfrak{s u}(2)$-valued connections on $M^{6}$ that cannot be traced back to lifts of connections on $M^{5}$. In contrast to e.g. [35], there are no instanton solutions that interpolate between these isolated instantons.

Remarks: First, the CY-limit $\Lambda \rightarrow \infty$ of (4.43) is given by

$$
\left[X_{a}, X_{b}\right]=f_{a b}{ }^{5}\left(X_{5}+\frac{1}{4} \frac{\mathrm{d}}{\mathrm{d} \tau} X_{5}\right)+f_{a b}{ }^{i} \mathcal{N}_{i} \quad \text { and } \quad\left[X_{5}, X_{a}\right]=f_{5 a}{ }^{b}\left(X_{b}+\frac{2}{3} \frac{\mathrm{d}}{\mathrm{d} \tau} X_{b}\right)
$$

wherein one requires the rescaling $X_{\mu} \mapsto \frac{1}{r} X_{\mu}$, which can be seen from $X_{\mu} e_{s}^{\mu} \rightarrow X_{\mu} r e^{\mu}$ for $\Lambda \rightarrow \infty$. Further, recall that in the limit $\Lambda \rightarrow \infty$ we have $\mathrm{d} \tau=\frac{1}{r} \mathrm{~d} r$. The above matrix equations coincide with the ones obtained in Kähler-torsion case of [53] as well as with the limit (4.30) of subsection 4.3.1. Remarkably, the two reductions of subsections 4.3.1 and 4.3.3 used the different $\mathfrak{s u}(2)$-instantons $\Gamma^{P}$ and $\Gamma_{\mathfrak{s u}(2)}$ as starting point; however, in the above limit the difference

$$
\Gamma^{P}-\Gamma_{\mathfrak{s u}(2)} \stackrel{\Lambda \rightarrow \infty}{\longrightarrow} \mathbb{1} \otimes \frac{\mathrm{d} r}{r} \in \Omega^{1}\left(M^{6}, \operatorname{End}\left(\mathbb{R}^{6}\right)\right)
$$

becomes an abelian flat part, which contributes to the instanton equation via the altered torsion.

Second, note the explicit impact of the conical singularities at $\varphi=0$ or $\varphi=\pi$ in the matrix equations (4.43) as well as the $B_{\mu}$-matrices of (4.40). However, we do not have to consider these singularities, as there is no well-defined instanton equation. 


\subsubsection{Transfer of solutions}

The previous subsections considered the nearly Kähler sine-cone from two perspectives: in subsection 4.3.1 we extended the instanton $\Gamma^{P}$, which is a connection on $\mathcal{Q}$; whereas, subsection 4.3.3 was concerned with $\Gamma_{\mathfrak{s u}(2)}$, being an $\mathfrak{s u}(2)$-valued connection on $\mathcal{Q}^{\prime}$, as a starting point for our ansatz (4.5). The local representations of these are related via a transformation $L$ as considered in (4.35). Due to the properties of $L$ we arrive at the following statement (cf. subsection 4.2):

$$
e^{\prime *} \Gamma_{\mathfrak{s u}(2)}=e^{\prime *} \Gamma^{P}-L^{-1} \mathrm{~d} L=e^{*} \Gamma^{P},
$$

implying that $\Gamma_{\mathfrak{s u}(2)}$ and $\Gamma^{P}$ have the same components with respect to their adapted coframes $e^{\prime}$ and $e$. Observe that the inhomogeneous part that is split off in the connection 1-form enters in the torsion (4.42) of $\Gamma_{\mathfrak{s u}(2)}$, thus altering the matrix equations. However, from (4.13) one can check that the local expressions of the respective field strengths of the extension of both $\Gamma^{P}$ and $\Gamma_{\mathfrak{s u}(2)}$ by $X_{\mu} \otimes e^{\prime \mu}=X_{\mu} L_{\nu}^{\mu} \otimes e^{\nu}$ coincide. Consequently, every instanton extension $X_{\mu}$ of $\Gamma_{\mathfrak{s u}(2)}$ gives rise to an instanton extension $X_{\nu} L_{\mu}^{\nu}$ of $\Gamma^{P}$ and vice versa. In other words, we have the relation

$$
X_{\mu} \text { solves }(4.43) \stackrel{1: 1}{\Longleftrightarrow} X_{\nu} L_{\mu}^{\nu} \text { solves }(4.24) \text {. }
$$

As a remark, the above is true if and only if $L$ takes values in the centralizer $C_{\mathrm{GL}(6, \mathbb{R})}(\mathrm{SU}(2))$, as then $L^{-1} \mathrm{~d} L$ gives rise to a well-defined equivariant 1-form.

However, one should not naively expect that the solutions obtained in subsections 4.3.1 and (4.3.3) are related via (4.52), as this does not necessarily transform the employed ansätze into one another.

The benefit from observation (4.52) is that we can generate further instanton solutions from our previous ones.

On the one hand, we can apply the above to (4.28) and obtain the ansatz

$$
X_{a}=\frac{\psi(r)}{\Lambda \sin \left(\frac{r}{\Lambda}\right)}\left(\exp \left(\frac{r}{2 \Lambda} \eta^{2}\right) \exp \left(\xi \eta^{3}\right)\right)_{a}^{b} \hat{I}_{b} \quad \text { and } \quad X_{5}=\frac{\chi(r)}{\Lambda \sin \left(\frac{r}{\Lambda}\right)} \hat{I}_{5}
$$

which inserted into (4.43) has precisely the solutions $(\psi, \chi)=(0,0),( \pm 1,1)$, just as one would expect from the above arguments. This is another non-constant instanton extension for $\Gamma_{\mathfrak{s u}(2)}$.

On the other hand, the same can be done for (4.45) in the other direction. There one derives the ansatz

$$
X_{a}=\psi(r) \Lambda \sin \left(\frac{r}{\Lambda}\right) \exp \left(-\frac{r}{2 \Lambda} \eta^{2}\right)_{a}^{b} B_{b}(r) \quad \text { and } \quad X_{5}=\chi(r) \Lambda \sin \left(\frac{r}{\Lambda}\right) B_{5}(r)
$$


Rewritten in a linear combination of the $\hat{I}_{\mu}$, the ansatz (4.54) is given as

$$
\begin{aligned}
& X_{1}=\psi(r)\left(\cos ^{3}\left(\frac{r}{2 \Lambda}\right) \hat{I}_{1}-\sin ^{3}\left(\frac{r}{2 \Lambda}\right) \hat{I}_{3}\right), \\
& X_{2}=\psi(r)\left(\cos ^{3}\left(\frac{r}{2 \Lambda}\right) \hat{I}_{2}+\sin ^{3}\left(\frac{r}{2 \Lambda}\right) \hat{I}_{4}\right), \\
& X_{3}=\psi(r)\left(\cos ^{3}\left(\frac{r}{2 \Lambda}\right) \hat{I}_{3}+\sin ^{3}\left(\frac{r}{2 \Lambda}\right) \hat{I}_{1}\right), \\
& X_{4}=\psi(r)\left(\cos ^{3}\left(\frac{r}{2 \Lambda}\right) \hat{I}_{4}-\sin ^{3}\left(\frac{r}{2 \Lambda}\right) \hat{I}_{2}\right), \\
& X_{5}=\chi(r) \cos \left(\frac{r}{\Lambda}\right) \hat{I}_{5} .
\end{aligned}
$$

One can check that this, again, produces the solutions $(\psi, \chi)=(0,0),( \pm 1,1)$. Remarkably, the two non-trivial instanton solutions correspond to non-constant extensions of $\Gamma^{P}$.

\subsection{Instantons on half-flat cylinders}

Let us now return to the half-flat 6-manifolds constructed in section 3.4 and apply the ansatz developed above to the instanton equation on these spaces. The instanton equation on spaces with non-vanishing $W_{2}$ was introduced in (4.3). In a local coframe adapted to the $\mathrm{SU}(3)$-structure imposing the pseudo-holomorphicity condition

$$
\Omega_{z} \wedge \mathcal{F}=0
$$

yields the set of six equations, precisely as it has been in the nearly Kähler case. But the additional equation implied by the pseudo-holomorphicity condition reads

$$
\mathrm{d} \Omega_{z} \wedge \mathcal{F}=0 \quad \Leftrightarrow \quad \mathcal{F}_{12}+\mathcal{F}_{34}+\frac{4}{3} \varrho^{2} \mathcal{F}_{56}=0
$$

in the rotated frame $e_{z}$. Note that for $\varrho= \pm \frac{\sqrt{3}}{2}$ this coincides with the nearly Kähler instanton equation of subsection 4.3.1, although the SU(3)-structure is not nearly Kähler (see for example the torsion classes (3.30)).

It is important to recall that the lift of the canonical connection of the Sasaki-Einstein $M^{5}$ provides an instanton on the cylinder that one can extend by some $X$ in our ansatz to $\mathfrak{s u}(3)$-valued connections, being defined either on $\mathcal{P}$ or $\mathcal{P}^{\prime}$. We will do so in two setups: first, we formulate the matrix equations in the frame $e^{\mu}$ and, second, the analogous computation is performed in the adapted frame $e_{z}^{\mu}$ for the half-flat $S U(3)$-structure.

\subsubsection{Matrix equations — Part I}

In the unrotated frame $e^{\mu}$ the instanton bundle is locally spanned by

$$
e^{5} \wedge e^{6}-\frac{\varrho}{3}\left(\cos \zeta \eta_{a b}^{1}-\sin \zeta \eta_{a b}^{2}\right) e^{a} \wedge e^{b} \quad \text { and } \quad e^{a} \wedge e^{6}-\varrho\left(\cos \zeta \eta_{b}^{1 a}-\sin \zeta \eta_{b}^{2 a}\right) e^{b} \wedge e^{5},
$$

from which we can extract the components of $\left(N_{\mu \nu}^{\rho}\right)$ to be

$$
N_{a b}^{5}=\frac{2 \varrho}{3}\left(\cos \zeta \eta_{a b}^{1}-\sin \zeta \eta_{a b}^{2}\right) \quad \text { and } \quad N_{b 5}^{a}=\varrho\left(\cos \zeta \eta_{b}^{1 a}-\sin \zeta \eta_{b}^{2 a}\right) \text {. }
$$


As the torsion components are unchanged we can directly formulate the matrix equations

$$
\begin{aligned}
{\left[\hat{I}_{i}, X_{\mu}\right] } & =f_{i \mu}{ }^{\nu} X_{\nu} \\
{\left[X_{a}, X_{b}\right] } & =\frac{2 \varrho}{3}\left(\cos \zeta \eta_{a b}^{1}-\sin \zeta \eta_{a b}^{2}\right) \dot{X}_{5}+2 \eta_{a b}^{3} X_{5}+f_{a b}{ }^{i} \mathcal{N}_{i} \\
{\left[X_{5}, X_{a}\right] } & =\varrho\left(\cos \zeta \eta_{a}^{1}{ }_{a}^{b}-\sin \zeta \eta_{a}^{2}{ }_{a}\right) \dot{X}_{b}+\frac{3}{2} \eta^{3}{ }_{a}^{b} X_{b} .
\end{aligned}
$$

The ansatz

$$
X_{a}=\psi(r)\left(\exp \left(\xi \eta^{3}\right)\right)_{a}^{b} \hat{I}_{b} \quad \text { for } \quad \xi \in[0,2 \pi) \quad \text { and } \quad X_{5}=\chi(r) \hat{I}_{5}
$$

satisfies, again, the equivariance condition of (4.60) and we obtain

$$
\mathcal{N}_{i}=\psi^{2}(r) \hat{I}_{i}, \quad \text { for } \quad i=6,7,8
$$

as well as the set of equations

$$
\dot{\psi}(r)=\dot{\chi}(r)=0, \quad \psi^{2}(r)=\chi(r), \quad \text { and } \quad \psi(r)(\chi(r)-1)=0 .
$$

for the two functions $\psi$ and $\chi$, and the equations hold for all values of $\xi$. Interestingly, the solutions to these equations are identical to the nearly Kähler case (4.27)

- $(\psi, \chi)=(\mathbf{0}, \mathbf{0})$ : the trivial solution appears again for consistency.

- $(\psi, \chi)=( \pm \mathbf{1}, \mathbf{1})$ : these two extensions of the lift of $\Gamma^{P}$ are newly obtained $\Omega_{z^{-}}$ instantons; however, they correspond to lifts of $M^{5}$-instantons because they are independent of the cylinder direction. Recall that $(\psi, \chi)=(-1,1)$ can be generated from $(\psi, \chi)=(+1,1)$ by the shift $\xi \mapsto \xi+\pi$.

Identically to the nearly Kähler case, one obtains the one-parameter family (4.28) as a solution.

As a matter of fact, these instanton solutions are identical to the ones obtained in subsection 4.3.1. The explanation is as follows: first, note that nearly Kähler 6-manifolds are a subset of half-flat 6-manifolds; thus, any nearly Kähler instanton solution must necessarily appear in the half-flat scenario. Second, the matrix equations (4.24) and (4.60) differ only in their derivative parts, i.e. in the coefficients of $\dot{X}_{\mu}$, which implies that both sets have coinciding constant solutions.

\subsubsection{Matrix equations - Part II}

Contrary to the previous subsection, here the focus is on the formulation of the instanton equations in the adapted coframe $e_{z}^{\mu}$ for the $\mathrm{SU}(3)$-structure on the cylinder. As with respect to these, the $\mathrm{SU}(3)$-structure forms have their standard components, one only has to compute the components of its torsion with respect to the transformed basis.

The space $\mathfrak{m}$ is now spanned by the 2 -forms

$$
e_{z}^{5} \wedge e_{z}^{6}-\frac{1}{3} \varrho^{2} \eta_{a b}^{3} e_{z}^{a} \wedge e_{z}^{b} \quad \text { and } \quad e_{z}^{a} \wedge e_{z}^{6}-\eta_{b}^{3 a} e_{z}^{b} \wedge e_{z}^{5},
$$


which follows from direct evaluation of (4.56) and (4.57). In the coframe $e_{z}$ the torsion components of the lifted canonical connection of the Sasaki-Einstein manifold are

$$
\tilde{T}_{a b}^{5}=2 \varrho \eta_{a b}^{1} \quad \text { and } \quad \tilde{T}_{b 5}^{a}=\frac{3}{2 \varrho} \eta_{b}^{1 a} .
$$

In addition, we need the tensor $N$ that appeared in (4.15). Since the instanton equations take a slightly different form here, its components now read

$$
N_{\mu \nu}^{a}=\frac{2}{3} f_{\mu \nu}^{a} \quad \text { and } \quad N_{\mu \nu}^{5}=\frac{1}{3} \varrho^{2} f_{\mu \nu}{ }^{5},
$$

wherein we have used the same $\mathfrak{s u}(3)$ structure constants as in (4.20). With these alterations (4.15) can be written as

$$
\begin{aligned}
{\left[\hat{I}_{i}, X_{\mu}\right] } & =f_{i \mu}{ }^{\nu} X_{\nu}, \\
{\left[X_{a}, X_{b}\right] } & =-2 \varrho \eta_{a b}^{1} X_{5}+\frac{2}{3} \varrho^{2} f_{a b}{ }^{5} \dot{X}_{5}+\mathcal{N}_{i} f_{a b}{ }^{i}, \\
{\left[X_{a}, X_{5}\right] } & =\frac{3}{2 \varrho} \eta_{a}^{1 b} X_{b}+\frac{2}{3} f_{a 5}{ }^{b} \dot{X}_{b} .
\end{aligned}
$$

One can employ the following ansatz:

$$
X_{a}=\psi(r)\left(\exp \left(\xi \eta^{1}\right) \exp \left(\theta \eta^{2}\right)\right)_{a}^{b} \hat{I}_{b}, \quad \text { for } \quad \theta, \xi \in[0,2 \pi) \quad \text { and } \quad X_{5}=\chi(r) \hat{I}_{5},
$$

which, again, satisfies the equivariance condition. The insertion of (4.68) into (4.67) yields for the $\mathfrak{s u}(2)$-part

$$
\mathcal{N}_{i}=\psi^{2} I_{i},
$$

as the projection of $\left[X_{a}, X_{b}\right]$ onto $\mathfrak{s u}(2)$ in $\mathfrak{s u}(3)$ is independent of $\theta$ and $\xi$. Further, for the functions $\psi$ and $\chi$ one derives the set of equations

$$
\begin{aligned}
\dot{\chi} & =\frac{3}{\varrho^{2}} \psi^{2}\left(\cos ^{2} \theta-\sin ^{2} \theta\right), \\
\chi & =\frac{2}{\varrho} \psi^{2} \cos \theta \sin \theta, \\
\dot{\psi} \cos \theta & =\frac{3}{2} \psi\left(\frac{1}{\varrho} \sin \theta+\chi \cos \theta\right), \\
\dot{\psi} \sin \theta & =-\frac{3}{2} \psi\left(\frac{1}{\varrho} \cos \theta+\chi \sin \theta\right) .
\end{aligned}
$$

Note that the equations are independent of $\xi$. These equations are mutually compatible only for $\theta=\frac{\pi}{4}$ or $\theta=\frac{3 \pi}{4}$. For these values of $\theta$ the first two equations yield $\dot{\psi}=\dot{\chi}=0$ and the last two equations coincide. The system (4.70) admits, besides the trivial solution $(\psi, \chi)=(0,0)$, only the following solutions:

$$
\begin{array}{ll}
\theta=\frac{\pi}{4}: \quad & \psi= \pm 1, \quad \chi=+\frac{1}{\varrho}, \\
\theta=\frac{3 \pi}{4}: & \psi= \pm 1, \quad \chi=-\frac{1}{\varrho} .
\end{array}
$$


Hence, we again have a whole family of solutions given by

$$
A=\Gamma+\left(\exp \left(\xi \eta^{1}\right) \exp \left(\theta \eta^{2}\right)\right)_{a}^{b} \hat{I}_{b} \otimes e_{z}^{a} \pm \frac{1}{\varrho} \hat{I}_{5} \otimes e_{z}^{5}, \quad \text { for } \quad \theta \in\left\{\frac{\pi}{4}, \frac{3 \pi}{4}\right\}, \xi \in[0,2 \pi) .
$$

As the corresponding instantons on the cylinder over $M^{5}$ do neither depend on the cone coordinate nor contain $\mathrm{d} r$, they are actually lifts of instantons on $M^{5}$, which live on the pull-back bundle of the SU(3)-bundle on the slices of the cylinder.

\section{Conclusions}

We investigated the geometry of cylinders, cones and sine-cones over 5-dimensional SU(2)manifolds. On the resulting 6-dimensional conical SU(3)-manifolds we formulated generalized instanton equations and reduced them to matrix equations via the ansatz (4.5). In particular, we focused on nearly Kähler and half-flat SU(3)-manifolds, whereas previous work [53] had dealt with the Kähler-torsion (KT) and hyper-Kähler-torsion (HKT) cases.

In particular, we constructed a nearly Kähler 6-manifold as a sine-cone over an arbitrary Sasaki-Einstein 5-manifold by means of a rotation of the SU(2)-structures on the slices. Employing the ansatz (4.22), the instanton equation was reduced to the set (4.24) of matrix equations, for which we found a family of non-trivial, but constant solutions. All of these correspond to lifts of $M^{5}$-instantons to $C_{s}\left(M^{5}\right)$. In addition, in subsection 4.2.2 we obtained an instanton solution on the manifold $C_{s}\left(M^{5}\right)$ by the construction of its $\mathfrak{s u}(3)$-valued canonical connection. We decomposed this connection $\Gamma_{\mathfrak{s u}(3)}$ into another $\mathfrak{s u}(2)$-valued instanton $\Gamma_{\mathfrak{s u}(2)}$ plus an additional part resembling the ansatz used before. Using this decomposition and, again, carrying the reduction of the instanton equation out, we obtained a set of four equations for two functions which parametrize the ansatz. Its three solutions, for which the scalar functions take certain constant values, correspond to three instantons on the nearly Kähler sine-cone that cannot be constructed as lifts of instanton connections on $M^{5}$. As a by-product, we explicitly confirmed the nearly Kähler canonical connection to be an instanton. In addition, observing a correspondence between the solutions, we transferred the solutions of the two cases to new $r$-dependent instanton extensions of $\Gamma^{P}$ as well as $\Gamma_{\mathfrak{s u}(2)}$. Remarkably, the extension found for $\Gamma^{P}$ does not seem to correspond to a lift of an instanton from $M^{5}$.

Furthermore, we introduced a two-parameter family of half-flat structures on the cylinder over a generic Sasaki-Einstein 5-manifold. Again employing the ansatz (4.5) on these cylindrical half-flat 6-manifolds, we were able to deduce the matrix equations (4.67) on the two local frames $e^{\hat{\mu}}$ and $e_{z}^{\hat{\mu}}$. Moreover, we provided families of constant, but non-trivial solutions. In that case, the instantons obtained this way do correspond to lifts of instantons on $M^{5}$.

It would be interesting to extend the methods presented here, i.e. the reduction of the instanton equation to matrix equations and the construction of higher-dimensional $G$ structure manifolds from lower-dimensional ones, to other scenarios that appear in sting theory. For example, in M-theory desirable (internal) manifolds are 7-dimensional and are endowed with a $G_{2}$-structure. Therefore, the study of certain $\mathrm{SU}(3)$-structures seems to 
be promising, as one could hope to a obtain interesting $G_{2}$-geometries as well as explicit instanton solutions via the procedures employed here.

Returning to the heterotic supergravity point of view, we expect that our solutions to the instanton equations can be lifted to full solutions of the heterotic equations of motions via the BPS equations (1.1) and the Bianchi identity (1.2). The gaugino equation (1.1c) is already solved by the instanton solutions above. The remaining equations should be solvable in a manner similar to [22, 23, 32, 33], which may look as follows:

1. The dilatino equation (1.1b) may be solved by a suitable ansatz such as choosing the dilaton $\phi=\phi(\tau)$ and the 3-form $H \propto \frac{\mathrm{d} \phi}{\mathrm{d} \tau} P$ where $P$ is the canonical 3-form on the Sasaki-Einstein 5-manifold.

2. The gravitino equation (1.1a) requires a spin connection with $\mathrm{SU}(3)$-holonomy and torsion $H$. Therefore, one can take an ansatz similar to (4.5) from which we know it to be an $\mathrm{SU}(3)$-instanton. The remaining task is then to check the correct torsion for this connection. One choice might be the canonical connection $\Gamma_{\mathfrak{s u}(3)}$ on the nearly Kähler sine-cone, whose torsion is by definition skew-symmetric and we know $\Gamma_{\mathfrak{s u}(3)}$ is an instanton.

3. The theorem of Ivanov requires a connection $\nabla$ on $T M^{6}$ which is an instanton. Here, the instantons constructed in this paper provide a valuable choice, i.e. by an extension of the canonical connection. Then the connection $\nabla$, together with the gauge connection $\mathcal{A}$, needs to satisfy the Bianchi identity (1.2).

Finally, one has to solve the differential equations that appear for the degrees of freedom in the different ansätze for $H, \nabla^{+}$, and $\nabla$. We hope to report on this process and embed our solutions into heterotic supergravity in the future.

\section{Acknowledgments}

This work was partially supported by the Deutsche Forschungsgemeinschaft grant LE $838 / 13$.

Open Access. This article is distributed under the terms of the Creative Commons Attribution License (CC-BY 4.0), which permits any use, distribution and reproduction in any medium, provided the original author(s) and source are credited.

\section{References}

[1] R. Rajaraman, Solitons and instantons. An introduction to solitons and instantons in quantum field theory, North-Holland, Amsterdam The Netherlands (1982).

[2] N. Manton and P. Sutcliffe, Topological solitons, Cambridge University Press, Cambridge U.K. (2004).

[3] E. J. Weinberg, Classical solutions in quantum field theory, Cambridge University Press, Cambridge U.K. (2012). 
[4] J. Polchinski, String theory. Volume 1: an introduction to the bosonic string, Cambridge University Press, Cambridge U.K. (1998).

[5] J. Polchinski, String theory. Volume 2: superstring theory and beyond, Cambridge University Press, Cambridge U.K. (1998).

[6] K. Becker, M. Becker and J.H. Schwarz, String theory and M-theory: a modern introduction, Cambridge University Press, Cambridge U.K. (2007).

[7] R. Harvey and H.B. Lawson Jr., Calibrated geometries, Acta Math. 148 (1982) 47.

[8] M. Graña, Flux compactifications in string theory: a comprehensive review, Phys. Rept. 423 (2006) 91 [hep-th/0509003] [INSPIRE].

[9] R. Blumenhagen, B. Körs, D. Lüst and S. Stieberger, Four-dimensional string compactifications with D-branes, orientifolds and fluxes, Phys. Rept. 445 (2007) 1 [hep-th/0610327] [INSPIRE].

[10] J. Louis and A. Micu, Heterotic string theory with background fluxes, Nucl. Phys. B 626 (2002) 26 [hep-th/0110187] [INSPIRE].

[11] J. Louis and A. Micu, Heterotic-type IIA duality with fluxes, JHEP 03 (2007) 026 [hep-th/0608171] [INSPIRE].

[12] G. Lopes Cardoso, G. Curio, G. Dall'Agata and D. Lüst, BPS action and superpotential for heterotic string compactifications with fluxes, JHEP 10 (2003) 004 [hep-th/0306088] [INSPIRE].

[13] A.R. Frey and M. Lippert, AdS strings with torsion: non-complex heterotic compactifications, Phys. Rev. D 72 (2005) 126001 [hep-th/0507202] [INSPIRE].

[14] P. Manousselis, N. Prezas and G. Zoupanos, Supersymmetric compactifications of heterotic strings with fluxes and condensates, Nucl. Phys. B 739 (2006) 85 [hep-th/0511122] [INSPIRE].

[15] K. Becker, M. Becker, K. Dasgupta and P.S. Green, Compactifications of heterotic theory on nonKähler complex manifolds. 1, JHEP 04 (2003) 007 [hep-th/0301161] [INSPIRE].

[16] K. Becker, M. Becker, P.S. Green, K. Dasgupta and E. Sharpe, Compactifications of heterotic strings on non-Kähler complex manifolds. 2, Nucl. Phys. B 678 (2004) 19 [hep-th/0310058] [INSPIRE].

[17] M. Fernandez, S. Ivanov, L. Ugarte and R. Villacampa, Non-Kähler heterotic string compactifications with non-zero fluxes and constant dilaton, Commun. Math. Phys. 288 (2009) 677 [arXiv: 0804.1648] [inSPIRE].

[18] G. Papadopoulos, Heterotic supersymmetric backgrounds with compact holonomy revisited, Class. Quant. Grav. 27 (2010) 125008 [arXiv:0909. 2870] [INSPIRE].

[19] J. Held, D. Lüst, F. Marchesano and L. Martucci, DWSB in heterotic flux compactifications, JHEP 06 (2010) 090 [arXiv: 1004.0867] [INSPIRE].

[20] O. Lechtenfeld, C. Nölle and A.D. Popov, Heterotic compactifications on nearly Kähler manifolds, JHEP 09 (2010) 074 [arXiv:1007.0236] [INSPIRE].

[21] M. Klaput, A. Lukas and C. Matti, Bundles over nearly-Kähler homogeneous spaces in heterotic string theory, JHEP 09 (2011) 100 [arXiv:1107.3573] [INSPIRE].

[22] D. Harland and C. Nölle, Instantons and Killing spinors, JHEP 03 (2012) 082 [arXiv:1109.3552] [INSPIRE]. 
[23] K.-P. Gemmer, A.S. Haupt, O. Lechtenfeld, C. Nölle and A.D. Popov, Heterotic string plus five-brane systems with asymptotic $\mathrm{AdS}_{3}$, Adv. Theor. Math. Phys. 17 (2013) 771 [arXiv: 1202.5046] [INSPIRE].

[24] C. Hull, Superstring compactifications with torsion and space-time supersymmetry, in $1^{\text {st }}$ Torino Meeting on Superunification and Extra Dimensions, P. Fre and R. D'Auria eds., World Scientific Publishing, Singapore (1986).

[25] C.M. Hull, Anomalies, ambiguities and superstrings, Phys. Lett. B 167 (1986) 51 [INSPIRE].

[26] C.M. Hull, Compactifications of the heterotic superstring, Phys. Lett. B 178 (1986) 357 [INSPIRE].

[27] A. Strominger, Superstrings with torsion, Nucl. Phys. B 274 (1986) 253 [inSPIRE].

[28] S. Ivanov, Heterotic supersymmetry, anomaly cancellation and equations of motion, Phys. Lett. B 685 (2010) 190 [arXiv:0908.2927] [INSPIRE].

[29] S.K. Donaldson, Anti self-dual Yang-Mills connections over complex algebraic surfaces and stable vector bundles, Proc. London Math. Soc. 50 (1985) 1.

[30] S.K. Donaldson, Infinite determinants, stable bundles and curvature, Duke Math. J. 54 (1987) 231.

[31] K. Uhlenbeck and S.T. Yau, On the existence of hermitian-Yang-Mills connections in stable vector bundles, Commun. Pure Appl. Math. 39 (1986) S257.

[32] A. Chatzistavrakidis, O. Lechtenfeld and A.D. Popov, Nearly Kähler heterotic compactifications with fermion condensates, JHEP 04 (2012) 114 [arXiv:1202.1278] [INSPIRE].

[33] K.-P. Gemmer and O. Lechtenfeld, Heterotic $G_{2}$-manifold compactifications with fluxes and fermionic condensates, JHEP 11 (2013) 182 [arXiv:1308.1955] [INSPIRE].

[34] M. Fernandez, S. Ivanov, V. Muñoz and L. Ugarte, Nearly hypo structures and compact nearly Kähler 6-manifolds with conical singularities, math/0602160 [INSPIRE].

[35] K.-P. Gemmer, O. Lechtenfeld, C. Nölle and A.D. Popov, Yang-Mills instantons on cones and sine-cones over nearly Kähler manifolds, JHEP 09 (2011) 103 [arXiv:1108.3951] [INSPIRE].

[36] B.S. Acharya, J.M. Figueroa-O'Farrill, C.M. Hull and B.J. Spence, Branes at conical singularities and holography, Adv. Theor. Math. Phys. 2 (1999) 1249 [hep-th/9808014] [INSPIRE].

[37] P. Koerber, D. Lüst and D. Tsimpis, Type IIA AdS $S_{4}$ compactifications on cosets, interpolations and domain walls, JHEP 07 (2008) 017 [arXiv:0804.0614] [INSPIRE].

[38] C. Nölle, Instantons, five-branes and fractional strings, arXiv:1207.7268 [INSPIRE].

[39] E. Corrigan, C. Devchand, D.B. Fairlie and J. Nuyts, First order equations for gauge fields in spaces of dimension greater than four, Nucl. Phys. B 214 (1983) 452 [INSPIRE].

[40] M. Mamone Capria and S.M. Salamon, Yang-Mills fields on quaternionic spaces, Nonlinearity 1 (1988) 517.

[41] R.R. Carrión, A generalization of the notion of instanton, Diff. Geom. Appl. 8 (1998) 1.

[42] L. Baulieu, H. Kanno, and I.M. Singer, Special quantum field theories in eight and other dimensions, Commun. Math. Phys. 194 (1998) 149 [hep-th/9704167] [INSPIRE]. 
[43] M. Blau and G. Thompson, Euclidean SYM theories by time reduction and special holonomy manifolds, Phys. Lett. B 415 (1997) 242 [hep-th/9706225] [INSPIRE].

[44] B.S. Acharya, J.M. Figueroa-O'Farrill, B.J. Spence and M. O'Loughlin, Euclidean D-branes and higher dimensional gauge theory, Nucl. Phys. B 514 (1998) 583 [hep-th/9707118] [INSPIRE].

[45] S. Donaldson and R. Thomas, Gauge theory in higher dimensions, in The geometric universe, S.A. Huggett et al. eds. Oxford University Press, Oxford U.K. (1996).

[46] S. Donaldson and E. Segal, Gauge theory in higher dimensions, II, in Surveys in differential geometry, N.C. Leung and S.-T. Yau eds., International Press of Boston, Boston U.S.A. (2009), arXiv:0902.3239 [INSPIRE].

[47] G. Tian, Gauge theory and calibrated geometry. 1, Annals Math. 151 (2000) 193 [math/0010015] [INSPIRE].

[48] D.B. Fairlie and J. Nuyts, Spherically symmetric solutions of gauge theories in eight-dimensions, J. Phys. A 17 (1984) 2867 [INSPIRE].

[49] S. Fubini and H. Nicolai, The octonionic instanton, Phys. Lett. B 155 (1985) 369 [InSPIRE].

[50] T.A. Ivanova and A.D. Popov, Selfdual Yang-Mills fields in $D=7,8$, octonions and Ward equations, Lett. Math. Phys. 24 (1992) 85 [InSPIRE].

[51] T.A. Ivanova and A.D. Popov, (Anti)selfdual gauge fields in dimension $d \geq 4$, Theor. Math. Phys. 94 (1993) 225 [INSPIRE].

[52] M. Günaydin and H. Nicolai, Seven-dimensional octonionic Yang-Mills instanton and its extension to an heterotic string soliton, Phys. Lett. B 351 (1995) 169 [Addendum ibid. B 376 (1996) 329] [hep-th/9502009] [INSPIRE].

[53] S. Bunk, T.A. Ivanova, O. Lechtenfeld, A.D. Popov and M. Sperling, Instantons on sine-cones over Sasakian manifolds, Phys. Rev. D 90 (2014) 065028 [arXiv:1407.2948] [INSPIRE].

[54] J. Sparks, Sasaki-Einstein manifolds, Surveys Diff. Geom. 16 (2011) 265 [arXiv:1004.2461] [INSPIRE].

[55] C.P. Boyer and K. Galicki, Sasakian geometry, Oxford University Press, Oxford U.K. (2008).

[56] D. Harland, T.A. Ivanova, O. Lechtenfeld and A.D. Popov, Yang-Mills flows on nearly Kähler manifolds and $G_{2}$-instantons, Commun. Math. Phys. 300 (2010) 185 [arXiv: 0909.2730] [INSPIRE].

[57] D. Conti and S. Salamon, Generalized Killing spinors in dimension 5, Trans. Amer. Math. Soc. 359 (2007) 5319 [math/0508375].

[58] F. Müller-Hoissen and R. Stückl, Coset spaces and ten-dimensional unified theories, Class. Quant. Grav. 5 (1988) 27 [INSPIRE].

[59] S. Chiossi and S. Salamon, The intrinsic torsion of $\mathrm{SU}(3)$ and $G_{2}$ structures, J. Diff. Geom. (2002) [math/0202282] [INSPIRE].

[60] T. Friedrich and S. Ivanov, Parallel spinors and connections with skew symmetric torsion in string theory, Asian J. Math 6 (2002) 303 [math/0102142] [INSPIRE].

[61] J.-M. Bismut, A local index theorem for non Kähler manifolds, Math. Ann. 284 (1989) 681 
[62] D. Harland and A.D. Popov, Yang-Mills fields in flux compactifications on homogeneous manifolds with SU(4)-structure, JHEP 02 (2012) 107 [arXiv: 1005.2837] [INSPIRE].

[63] G. Lopes Cardoso et al., NonKähler string backgrounds and their five torsion classes, Nucl. Phys. B 652 (2003) 5 [hep-th/0211118] [INSPIRE].

[64] T.A. Ivanova and A.D. Popov, Instantons on special holonomy manifolds, Phys. Rev. D 85 (2012) 105012 [arXiv: 1203.2657] [INSPIRE].

[65] S. Kobayashi and K. Nomizu, Foundations of differential geometry, volume 2, reprint of the 1969 original, John Wiley \& Sons, Inc., New York U.S.A. (1996).

[66] D. Kapetanakis and G. Zoupanos, Coset space dimensional reduction of gauge theories, Phys. Rept. 219 (1992) 4 [INSPIRE]. 